Florida International University FIU Digital Commons

11-4-1999

\title{
A study of cartenoids uptake in human subjects and their metabolites
}

Yin Chen

Florida International University

DOI: $10.25148 /$ etd.FI14060180

Follow this and additional works at: https://digitalcommons.fiu.edu/etd

Part of the Chemistry Commons

\section{Recommended Citation}

Chen, Yin, "A study of cartenoids uptake in human subjects and their metabolites" (1999). FIU Electronic Theses and Dissertations. 2148.

https://digitalcommons.fiu.edu/etd/2148

This work is brought to you for free and open access by the University Graduate School at FIU Digital Commons. It has been accepted for inclusion in FIU Electronic Theses and Dissertations by an authorized administrator of FIU Digital Commons. For more information, please contact dcc@fiu.edu. 
FLORIDA INTERNATIONAL UNIVERSITY

Miami, Florida

\title{
A STUDY OF CAROTENOIDS UPTAKE IN HUMAN SUBJECTS AND THEIR METABOLITES
}

\author{
A thesis submitted in partial fulfillment of the \\ requirements for the degree of \\ MASTER OF SCIENCE \\ in \\ CHEMISTRY \\ by \\ YIN CHEN
}

1999 
To: Dean Arthur W. Herriott

College of Arts and Sciences

This thesis, written by Yin Chen, and entitled A Study of Carotenoids Uptake in Human Subjects and Their Metabolites, having been approved in respect to style and intellectual content, is referred to you for judgment.

We have read this thesis and recommend that it be approved.

Richard A. Bone

David Becker

John T. Landrum, Major Professor

Date of Defense: November 4, 1999

The thesis of Yin Chen is approved.

Dean Arthur W. Herriott College of Arts and Sciences

Dean Richard L. Campbell Division of Graduate Studies

Florida International University, 1999 


\section{DEDICATION}

I dedicate this thesis to my parents, my husband and especially my two wonderful sons, Peter and Eric. Without their patience, understanding, support, and most of all love, the completion of this work would not have been possible. 


\section{ACKNOWLEDGMENTS}

I wish to thank my committee members: Dr. Richard A. Bone, Dr. David Becker, and Dr. John T. Landrum for their support, patience, and good humor. Their gentle but firm direction has been most appreciated. Dr. Richard Bone has been patiently helping me in every detail while I was working in his lab. Dr. David Becker has given me a lot of good suggestions. Finally, I would especially like to thank my major professor, Dr. John T. Landrum for having the confidence in my abilities to complete this work.

And finally, I would like to thank Chritian Herrero and Mark Kilburn for assisting me with a lot of details. I also thank all the co-workers in the lab. 
ABSTRACT OF THE THESIS

\section{A STUDY OF CAROTENOIDS UPTAKE IN HUMAN SUBJECTS AND THEIR}

\section{METABOLITES}

by

Yin Chen

Florida International University, 1999

Miami, Florida

Professor John T. Landrum, Major Professor

In this study, a metabolite of lutein was tentatively shown to result from oxidation of lutein by performing a reduction reaction. The result is important evidence that supports the hypothesis that lutein functions as an antioxidant in the human body. In a long term of dietary supplementary study of zeaxanthin in a human subject, the expected increase in the serum concentration of the supplement was accompanied by an increase in serum concentration of several metabolites. A six-month low dose dietary supplementation with lutein in 22 human subjects was performed with an expected result in increase of the serum concentration lutein and macular pigment optical densities. The mean increase of serum lutein concentration and macular pigment optical density in all subjects are $128 \%$ and $4.5 \%$, respectively. The abundance of zeaxanthin stereoisomers in 18 of the subject's serum was also measured. We noted a significant difference in zeaxanthin stereoisomer composition between the serum and the retina. The all-trans-RR-zaexanthin was the predominate form presented in human serum. This result is in agreement with previously published work. 


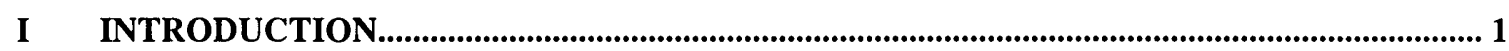

1. NATURAL OCCURRENCE AND DISTRIBUTION ........................................................................ 1

2. STRUCTURES AND PROPERTIES ……………………………………………………… 1

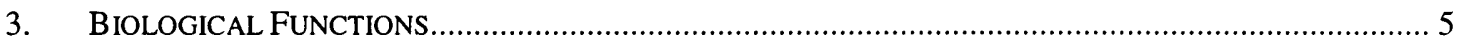

4. TECHNIQUES USED IN THE CAROTENOID ANALYSIS. …………………………………………... 7

5. OUTLINE OF EXPERIMENTS ................................................................................................ 10

II PART 1 STUDY OF THE METABOLITES OF CAROTENOIDS................................................ 11

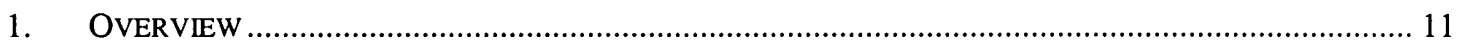

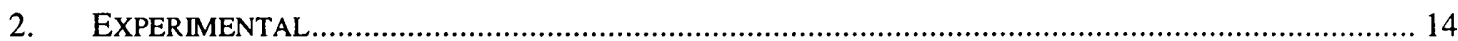

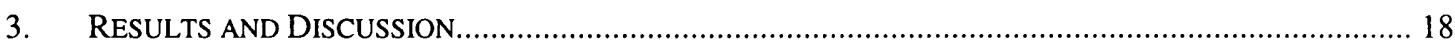

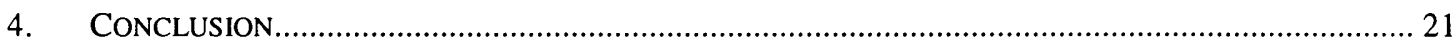

II PART 2 ZEAXANTHIN SUPPLEMENTATION STUDY …...................................................... 34

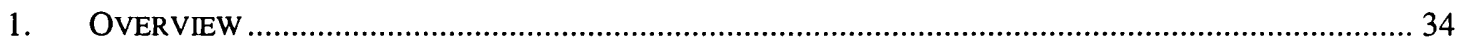

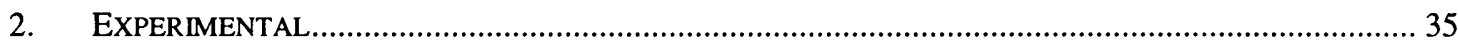

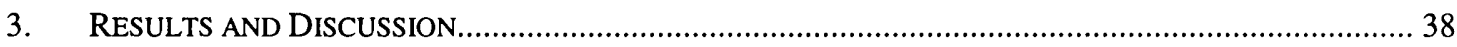

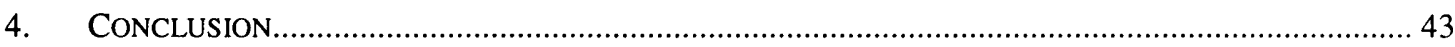

III PART 3 A SIX MONTH STUDY OF LUTEIN UPTAKE IN 22 HUMAN SUBJECTS ............. 45

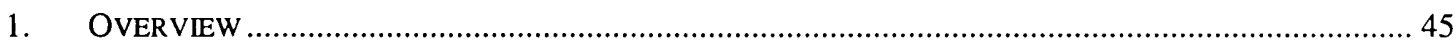

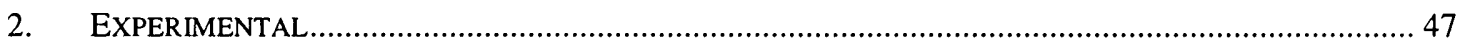

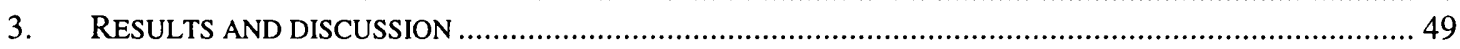

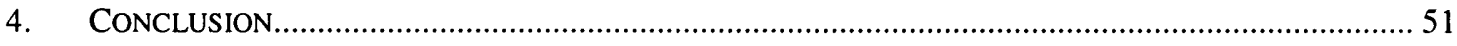

IV PART 4 ZEAXANTHIN STEREOISOMERS IN HUMAN SERUM SAMPLES ....................... 55

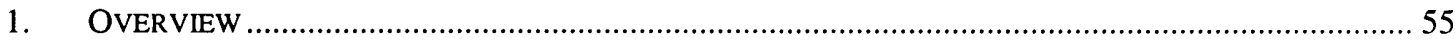

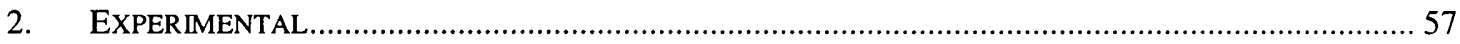

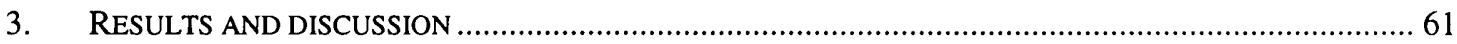

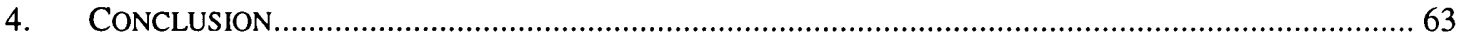

VI LIST OF REFERENCES .................................................................................................................... 65 


\section{LIST OF TABLES}

TABLES

PAGE

Table 1. Changes in peak ratio of Lutein peak/A and Lutein peak/B .19

Table 2. Serum average concentration and standard deviation of zeaxanthin and the metabolite A, B and C.

Table 3. Serum average concentration and standard deviation of zeaxanthin and the metabolites A, B and C

Table 4. The calculated half-lives for the uptake and decay of the concentration of lutein, zeaxanthin and the metabolites $\mathrm{B}$ and $\mathrm{C}$

Table 5. Pre- and post-supplementation serum lutein levels and macular pigment optical densities of the subjects

Table 6. Percent average increase from all human subjects in several carotenoids metabolites and optical density after supplementation

Table 7. Percentage of zeaxanthin stereoisomers in serum 62 


\section{LIST OF FIGURES}

FIGURES

PAGE

Figure 1. The structures of the most abundant carotenoids in human body..........2

Figure 2. Overlaping of hydrogen atoms and of hydrogen and methyl group with Zconfiguration.

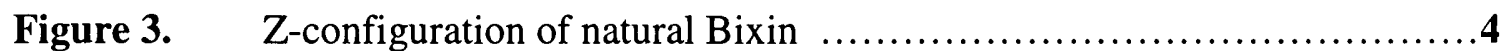

Figure 4. Comparison of the absorption spectra of geometrical isomers of $\beta, \beta-$ carotene in hexane..................................................................8

Figure 5. Equilibrium between lutein, 3'-Epilutein, and zeaxanthin in human serum (Khachik, et al. 1995)

Figure 6. Formation of diketocarotenoids from oxoluteins (Khachik, et al. 1995)...13

Figure 7. Typical HPLC chromatogram of carotenoids from serum extract $\ldots \ldots \ldots .23$

Figure 8. HPLC chromatogram of carotenoids from reduced serum extract.......23

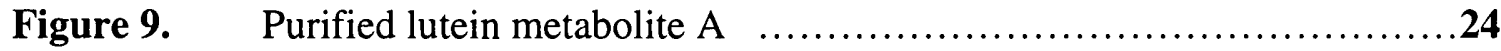

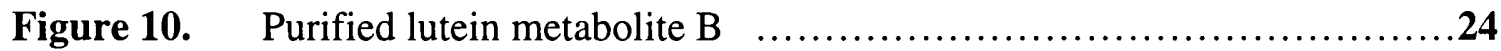

Figure 11. HPLC chromatogram of reduced lutein metabolite A $\ldots \ldots \ldots \ldots \ldots \ldots .25$

Figure 12. HPLC chromatogram of reduced lutein metabolite $B \quad \ldots \ldots \ldots \ldots \ldots \ldots 25$

Figure 13. HPLC chromatogram of Lutein/Zeaxanthin $(1: 1)$ standard $\ldots \ldots \ldots \ldots \ldots .26$

Figure 14. HPLC chromatogram of co-injection peak $A_{2}$ with Lutein/Zeaxanthin

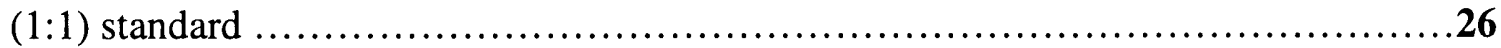

Figure 15. HPLC chromatogram of co-injection peak $B_{3}$ with Lutein/Zeaxanthin (1:1) standard

Figure 16. HPLC chromatogram of carotenoids from serum extract on a $10.00 \mathrm{~mm}$ C18 column 
Figure 17. UV/Vis spectrum of authentic lutein

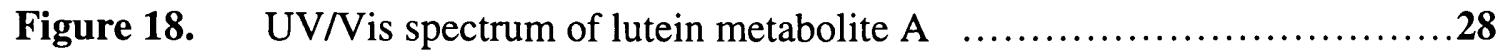

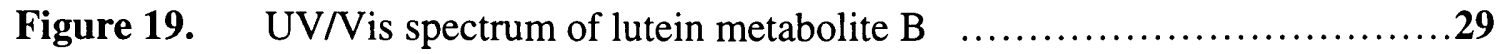

Figure 20. HPLC chromatogram of reduced lutein metabolite A on a $10.00 \mathrm{~mm} \mathrm{C18}$ column

Figure 21. HPLC chromatogram of reduced lutein metabolite B on a $10.00 \mathrm{~mm} \mathrm{C18}$ column..........................................................................

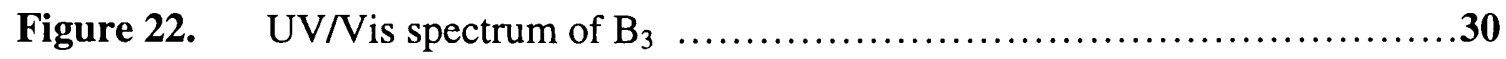

Figure 23. HPLC chromatogram of Lutein/Zeaxanthin (1:1) standard on a $10.00 \mathrm{~mm}$ C18 column......................................................................

Figure 24. HPLC chromatogram of co-injection peak $A_{2}$ with Lutein/Zeaxanthin (1:1) standard on a $10.00 \mathrm{~mm}$ C18 column........................................

Figure 25. HPLC chromatogram of co-injection peak $B_{3}$ with Lutein/Zeaxanthin (1:1) standard on a $10.00 \mathrm{~mm} \mathrm{C18} \mathrm{column.}$

Figure 26. UV/Vis spectrum of unknown peak from the chromatogram of reduced lutein metabolite $\mathrm{A}, \lambda_{\max }=452 \mathrm{~nm}$ 32

Figure 27. UV/Vis spectrum of unknown peak from the chromatogram of reduced

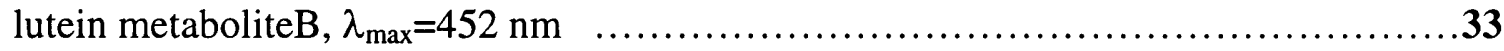

Figure 28. The structure of MHL. R=hexyl...............................38

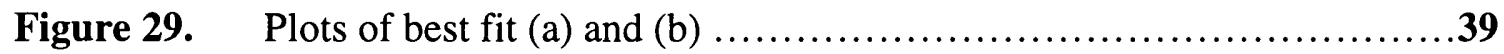

Figure 30. HPLC chromatogram of carotenoids from serum extract before supplementation (a), and after 40 days supplementation (b) $\ldots \ldots \ldots \ldots \ldots \ldots \ldots \ldots \ldots \ldots$

Figure 31. The plot of serum concentration of metabolite $A$ as a function of time...43

Figure 32. The plot of serum concentration of metabolite $B$ as a function of time...43

Figure 33. The plot of serum concentration of metabolite $C$ as a function of time...44

Figure 34. Plot of pre-supplementation serum lutein level vs dietary intake .52 
Figure 35. Plot of pre- supplementation vs post-supplementation serum lutein 53

Figure 36. The plots of serum lutein concentration as a function of time with lower baseline level (a) and higher baseline level 54

Figure 37. Stereochemical structures of zeaxanthin .55

Figure 38. Cross-sectional diagram of the human eye. (Handelman. 1992) .57

Figure 39. The chemical reaction of zeaxanthin dicabamate derivation .60

Figure 40. HPLC chromatogram of dicabamated zeaxanthin racemic-mixture standard on a normal phase HPLC

Figure 41. HPLC chromatogram of dicabamated zeaxanthin from serum extract on a normal phase HPLC .64

Figure 42. HPLC chromatogram of co-injection collected peak in a dicabamated zeaxanthin from serum extract with dicabamated zeaxanthin racemic-mixture standard on a normal phase HPLC 64 


\section{INTRODUCTION}

\section{Natural Occurrence and Distribution}

Carotenoids are one of the main classes of natural pigments. Around 600 naturally occurring carotenoids have been isolated and identified. (Straub, 1987) They are related to the beautiful colors of many fruits (e.g. pineapples, citrus, orange, tomatoes), vegetables (paprika, spinach and many green vegetables) and flowers (e.g. marigold, sunflower). The greatest production of carotenoids occurs in the photosynthetic tissues of plants, algae and bacteria. (Pfander, 1992) Although carotenoids are commonly considered plant pigments, they also occur widely in microorganisms, such as bacteria. Many animals are also colored by carotenoids, including birds (flamingo, ibis, canary), insects (ladybird) and marine animals (salmon). Significantly, carotenoids are synthesized in nature only by plants and microorganisms. Animals are not able to synthesize carotenoids. The carotenoids found in animals are derived from dietary sources.

\section{Structures and Properties}

As is well known, the carotenoid pigments belong to the class of polyenes. Most naturally occurring carotenoids contain 40 carbon atoms, corresponding to 8 isoprene units linked to form a conjugated double bond system. The carbon skeleton of the carotenoids is highly branched. The acyclic hydrocarbon lycopene, (Figure 1) can be considered as the prototype of these carotenoids. Other carotenoids can be considered to be related to lycopene through structural changes and as oxygenated derivatives. For example, $\beta, B$-carotene (Figure 1) can be regarded as resulting from cyclization of both 


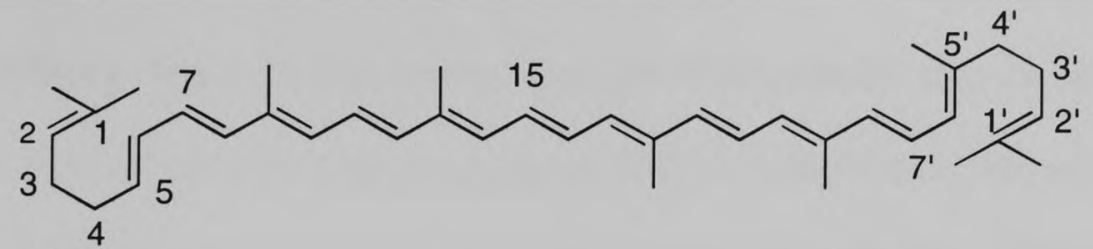

End-group

Lycopene

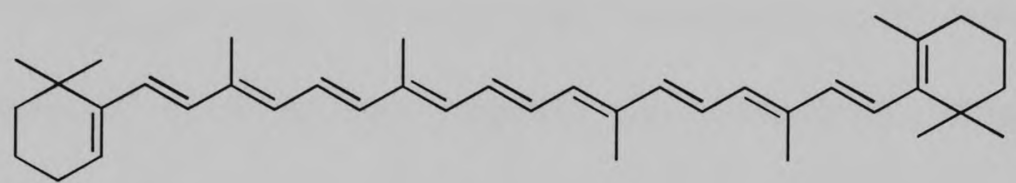

$\beta$-ring

$\beta, \beta$-Carotene<smiles>CC1=C(/C=C/C(C)=C/C=C/C(C)=C/C=C/C=C(C)/C=C/C=C(C)/C=C/C2=C(C)CC(O)CC2(C)C)C(C)(C)CC(O)C1</smiles>

3-Hydroxy- $\beta$-ring

\section{Zeaxanthin}<smiles>CC1=CC(O)CC(C)(C)C1/C=C/C(C)=C/C=C/C(C)=C/C=C/C=C(C)/C=C/C=C(C)/C=C/C1=C(C)CC(O)CC1(C)C</smiles>

\section{Lutein}

Figure 1. The structures of the most abundant carotenoids in human body 
end-groups in lycopene. The end-groups of carotenoids can form several different structural moieties. Since the two terminal groups of carotenoids may be either identical or different, both symmetrical and non-symmetrical carotenoid structures exist. The long, conjugated double-bond system also offers many spatial possibilities. The double bonds of the natural carotenoids are usually found to have an all-E (trans) geometry because this is the most stable geometry. The oxygenated carotenoids can be alcohols, aldehydes, ketones, or acids. Some of the most important and characteristic carotenoids are the carotenes lycopene, $\beta, \beta$-carotene, and the xanthophylls zeaxanthin, and lutein. Their structures are shown in Figure 1. They are the most abundant carotenoids in the human body because of their high concentration in dietary sources. (Khachik, et al., 1992A; Barua, et al., 1992; Khachik, et al., 1992B) The function and metabolism of those carotenoids in the human body is an area of considerable research activity.

Carotenoids have special properties due to their chemical structures. Because of the presence of the polyene chain, the all-trans or all-E carotenoids may undergo geometrical isomerization producing cis-or Z-isomers. A large number of geometrical isomers is theoretically possible for any carotenoid. (Zechmeister, 1962) In general, there are two types of double bonds in the polyene chain of most C40 carotenoids. (see Figure 2) However, the cis-geometry is sufficiently stable only for those double bonds which are of the type A where two $\mathrm{CH}$ groups are adjacent. This is because there is less hindrance from overlapping of hydrogen atoms in these bonds than those disubstituted double bonds of type B having a hydrogen atom and a methyl group which sterically interfere. It is not surprising that most natural carotenoids occur predominantly in the all-trans (all-E) form because its nearly planar structure allows for effective resonance throughout the polyene. 
An all-trans (all-E) carotenoid is generally expected to surpass in stability its cis-isomers (Z-isomers). In practice, few cis-isomers of carotenoids are encountered. Despite this lack of stability, a few examples of naturally abundant cis-isomers are known, for example natural Bixin, Figure 3.



Figure 3. Z-configuration of natural Bixin

Carotenoids are insoluble in water but are soluble in fats or in dipolar solvents. They are sensitive to light and heat which can cause E/Z isomerization, and to air and peroxides which can cause oxidation. In the presence of acid they can undergo addition reactions of electrophiles to the carotenoid. 


\section{Biological Functions}

The most characteristic feature of carotenoids is their observed color, which is caused by the conjugated polyene chain. As natural pigments, carotenoids are widely used as additives, to provide attractive colors, in manufactured foods. Although synthetic carotenoids are now produced on a large scale for food coloration, natural extracts are still extensively used.

In photosynthetic systems, carotenoids have the important functions of lightharvesting and photo-protection. (Cogdell, 1985) The functions of carotenoids in photosynthesis depend on their location and orientation relative to the other components. In photosynthetic bacteria, carotenoids act as accessory light-harvesting pigments where they function by transferring their excitation energy to bacteriochlorophyll through singlet energy transfer. Studies have demonstrated that natural all-trans (E) carotenoids are involved in this process. (Koyama et al., 1990) In plants and algae, carotenoids in the reaction center are able to protect against photosensitized oxidation by quenching the excitation energy of the chlorophyll triplet state and singlet oxygen.

Vitamin A deficiency is a worldwide nutritional problem. Vitamin A deficiency in humans can cause blindness and premature death. Vitamin A also has important systemic functions in humans related to growth, health maintenance, and development. As a micronutrient, the best known function of carotenoids is the provitamin A activity. (Zechmeister, 1962) Particularly ß,ß-carotene, but also, carotenoids with an unsubstituted $\beta$-ionene ring such as $\alpha$-carotene, $\gamma$-carotene, and $\beta$-cryptoxanthin are sources of vitamin A. These $\mathrm{C} 40$ - carotenes are converted in the body into vitamin A and vitamin A derivatives. The mechanism of the cleavage is not established. Central cleavage by a 15 , 
15 '-dioxygenase enzyme has been proven but isolation of the enzyme has proven illusive. Eccentric cleavage of other double bonds also occurs. (Zechmeister, 1962) The complete nature of the conversion process is still far from clear.

Several studies have demonstrated that carotenoids act as antioxidants and may have an important protective action against several types of cancers. (Khachik, et al., 1995) This protective effect is not related to the formation of vitamin A. A number of studies have shown that consuming large quantities of fruits and vegetables which contain major dietary carotenoids such as lycopene or lutein, reduces the risk of cancer. Lycopene and lutein have high antioxidant activity. (Khachik, et al., 1995)

The nonprovitamin A carotenoids have other biological functions and activities. For example, Bone et al established that the macular pigment may play a protective role in the eye. (Bone, et al., 1985; Schalch, 1992) The human macular pigment is a mixture of two carotenoids, lutein and zeaxanthin. (Bone, et al., 1985) The most important function of these carotenoids is to provide the central retina with protection against damaging photochemical reactions initiated by absorption of blue light. Blue light has a short wavelength and it can induce the formation of reactive free radicals, triplet excited states, superoxide, and singlet oxygen within the retina. The macular pigments harmlessly absorbs blue light reducing the potential for damage to the delicate retinal tissues. In a recent study, Landrum et al found that the serum levels of lutein and the level of lutein in the macula of the human eye are both increased by long-term dietary supplementation. (Landrum, et al., 1997)

Considerable evidence supports the hypothesis that light-promoted damage to the retina is one of several possible factors contributing to the development of Age - Related 
Macular Degeneration (AMD), which occurs in about $20 \%$ of the population above the age of 65. (Landrum, et al., 1997; Landrum, et al., 1995)

\section{Techniques used in the carotenoid analysis.}

In order to accurately identify individual carotenoids, a number of techniques are required. Because of their long, conjugated double bond system, the carotenoids exhibit a strong absorption in the visible and UV region. The extinction coefficients of many carotenoids have been published and are widely used. (Davies, 1976; De Ritter, et al., 1981; Britton, 1985; and Kohler, 1995) Several important facts can be obtained from the spectrum.

1) The wavelength of maximum absorption provides structural information. As the length of the chromophore increases, the energy required to promote one of the $\pi$ bonding electrons to the excited state is reduced and the absorption occurs at a longer wavelength.

2) The intensity of absorption is related to the structure. The optical density or absorbance is a measure of the concentration of the carotenoids. The formation of $\mathrm{Z}$ or cis-isomers will cause a hypsochromic shift in $\lambda_{\max }$ and a lowered value of the extinction coefficient results.

3) Most carotenoid spectra are characterized by three overlapping peaks. The degree of overlap provides important information for identification. It is useful to calculate the value of $\% \mathrm{III} / \mathrm{II}$ for describing fine structure. (III is the peak height of the longest-wavelength absorption band/ II is that of the middle absorption band) (Figure 4) . 


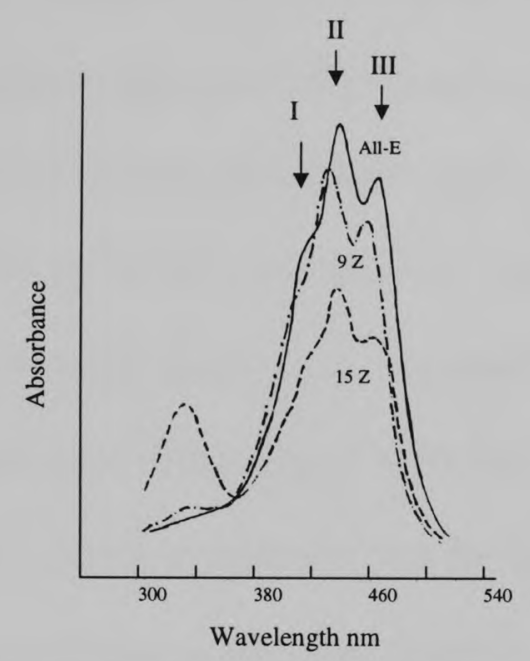

Figure 4. Comparison of the absorption spectra of geometrical isomers of $\beta, \beta$-carotene in hexane

4) The UV spectrum also provides some information to identify E/Z or cistrans isomers. In addition to the hypsochromic shift in the wavelength of maximum absorption, $\mathrm{Z}$ or cis-isomers exhibit a reduction in vibrational fine structure and the appearance of a cis peak at a position about $142 \mathrm{~nm}$ below the wavelength of maximum absorption. Usually the intensity of the cis-band is the greatest when the cis double bond is closest to the center of the molecule. Therefore, a 15-cis isomer shows a well-developed cis-band. (Figure 4) For conclusive identification, NMR is required.

Because of the involvement of carotenoids in many biochemical and biological processes, numerous methods of isolation and detection of carotenoids have been developed. HPLC in combination with a number of different detectors is the current 
method of choice. (Handelman, et al., 1992; Khachik, et al., 1992; and Craft, 1992)) HPLC with UV detection is the most frequently used and this method gives excellent separation in a short time and is highly sensitive. Alternatively, HPLC can be coupled with a multi-wavelength photodiode array detector for carotenoids analysis. (De Leenheer, et al., 1992; and Sowell, et al., 1988) In comparision to the UV detector, the photodiode array gives more information because complete spectra can be obtained which can be compared with those of standards. However, photodiode arrays are somewhat less sensitive than the conventional UV detectors and are more expensive. Identifications are often made by simply comparing retention times of unknown peaks with those of reference materials. However, only a limited number of synthetic carotenoid standards are readily available. It is unwise to attempt identifications based solely on retention time and UV spectrum since many carotenoids have very similar structures. Therefore, HPLC-MS coupling is another useful method. The specificity of mass spectrometric detection for identification of carotenoids greatly improves the identification process. (van Breemen, 1996; and van Breemen, et al., 1993)

The cis-isomers of cartenoids are generally poorly separated from trans-isomers. These isomers have sufficiently similar absorption coefficients such that the exact structure is often impossible to determine by UV techniques. HPLC-MS is also of limited usefulness for identification cis/trans isomers, all of which have the same mass, and yield identical fragmentation patterns. The method of choice for structural elucidation of cis/trans isomers is ${ }^{1} \mathrm{H}$ NMR spectroscopy. (Strohschein, et al., 1997) Large quantities (typically several $\mu \mathrm{g}$ ) are required for NMR. For this reason NMR is 
of limited value in practice due to the low concentrations of the carotenoids found in plants and other biological samples.

\section{Outline of experiments}

In order to address some of the problems that are of interest to the scientists working in the carotenoid field, my research work focuses on the following four topics:

Part 1 Study of Metabolites of Carotenoids

Part 2 Zeaxanthin Supplementation Study

Part 3 A Six months study of Lutein Uptake in 22 Human Subjects

Part 4 Zeaxanthin Stereoisomers in Human Serum Samples

Each part will contain an overview, experiment procedure, results and discussion, and conclusions. 


\section{PART 1 STUDY OF THE METABOLITES OF CAROTENOIDS}

\section{Overview}

The vitamin A activity of provitamin A carotenoids is well established. (Straub, 1987) However, the nutritional and metabolic benefits of non-provitamin A carotenoids are still insufficiently investigated. Recently, a number of epidemiological studies have demonstrated that a high consumption of fruits and vegetables may reduce the risk of cancer. (Khachik, et al., 1992; Khachik, et al., 1995; Khachik, et al., 1997; Khachik, et al., 1997) It was also reported that the chances of several types of cancer are very low in regions where consumption of several types of fruits and vegetables on part of the normal diet. (Khachik, et al., 1995) It is known that lutein, which is found in human serum, is a non-provitamin A carotenoid. (Khachik, et al., 1992; Barua, et al., 1992; Khachik, et al., 1992) Although the carotenoids found in commonly consumed fruits and vegetables have been identified, not all of them are present in human blood. It has been reported that at least 21 carotenoids are found in blood. Seven of these are reported to be metabolites. (Khachik, et al., 1995) Since the concentration levels of these metabolites are very low, their identification is very difficult. Khachik et al reported that oxidation products of lutein, zeaxanthin, and lycopene are present in human serum. (Khachik, et al., 1995) A possible metabolic pathway for the oxidation of lutein in humans was proposed. A combination of several reactions are required to account for the formation of these lutein metabolites. An equilibrium was suggested to exist between (3R,3'R,6'R)-lutein and (3R,3'R)-zeaxanthin and the intermediate (3R,3'S,6'R)-lutein, (3'-epilutein), as shown in 
Figure 5. (Khachick, et al., 1995) Allylic oxidation of natural lutein at the allylic 3' hydroxyl group and may result in the formation of oxolutein. It was suggested that the double bond migration followed by allylic oxidation of oxoluteins would result in the formation of mono- and di-ketocarotenoids as shown in Figure 6. (Khachick, et al., 1995) The proposed mechanism by which carotenoids function in cancer prevention is based on their antioxidant capability. They are able to quench singlet oxygen and react with other oxidizing species, inhibit lipid peroxidation, and thereby prevent promotion and replication in the neoplastic cells. Lutein is a proposed antioxidant and its oxidation products should be found in serum.

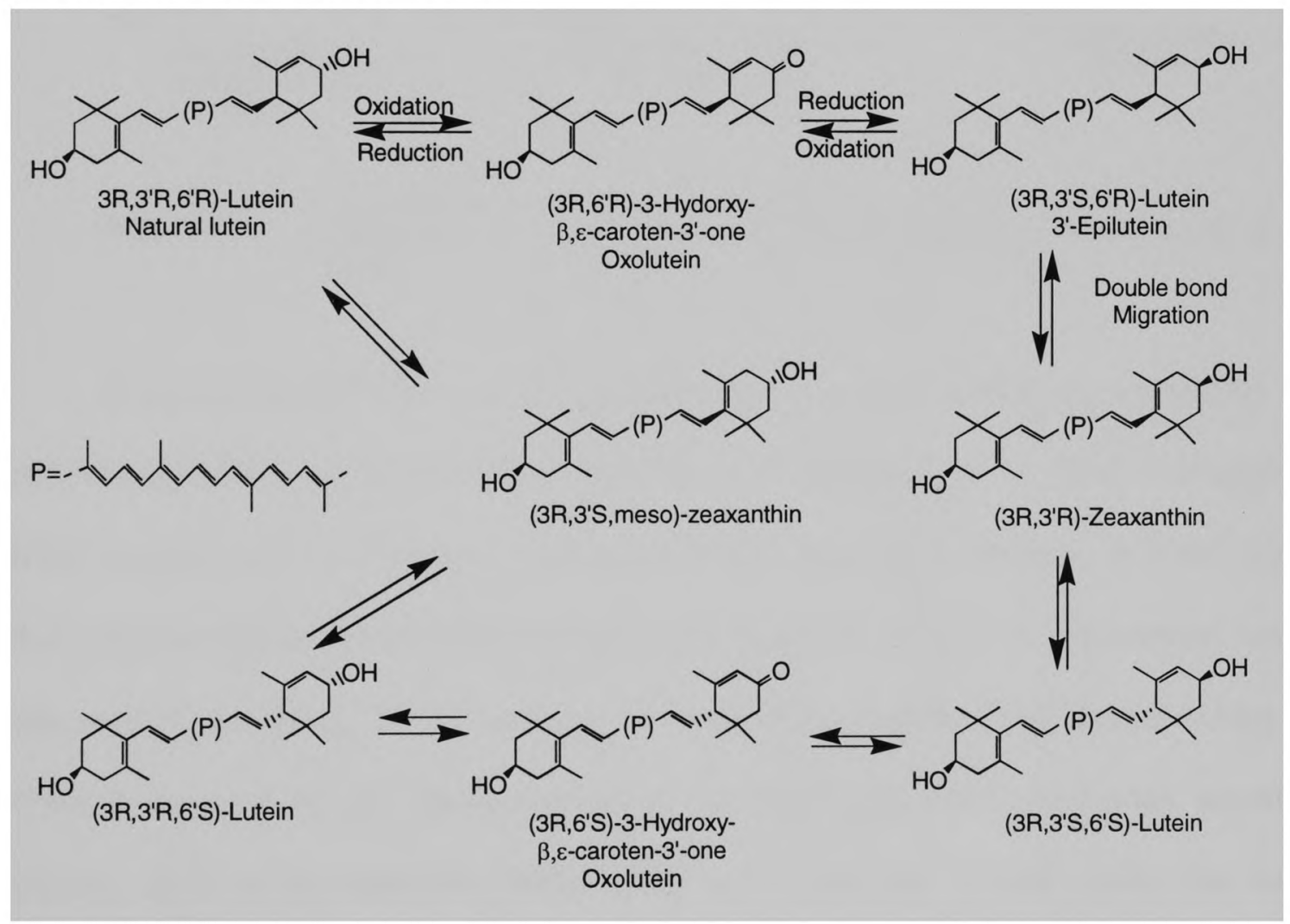

Figure 5. Equilibrium between lutein, 3'-Epilutein, and zeaxanthin in human serum (Khachik, et al. 1995) 


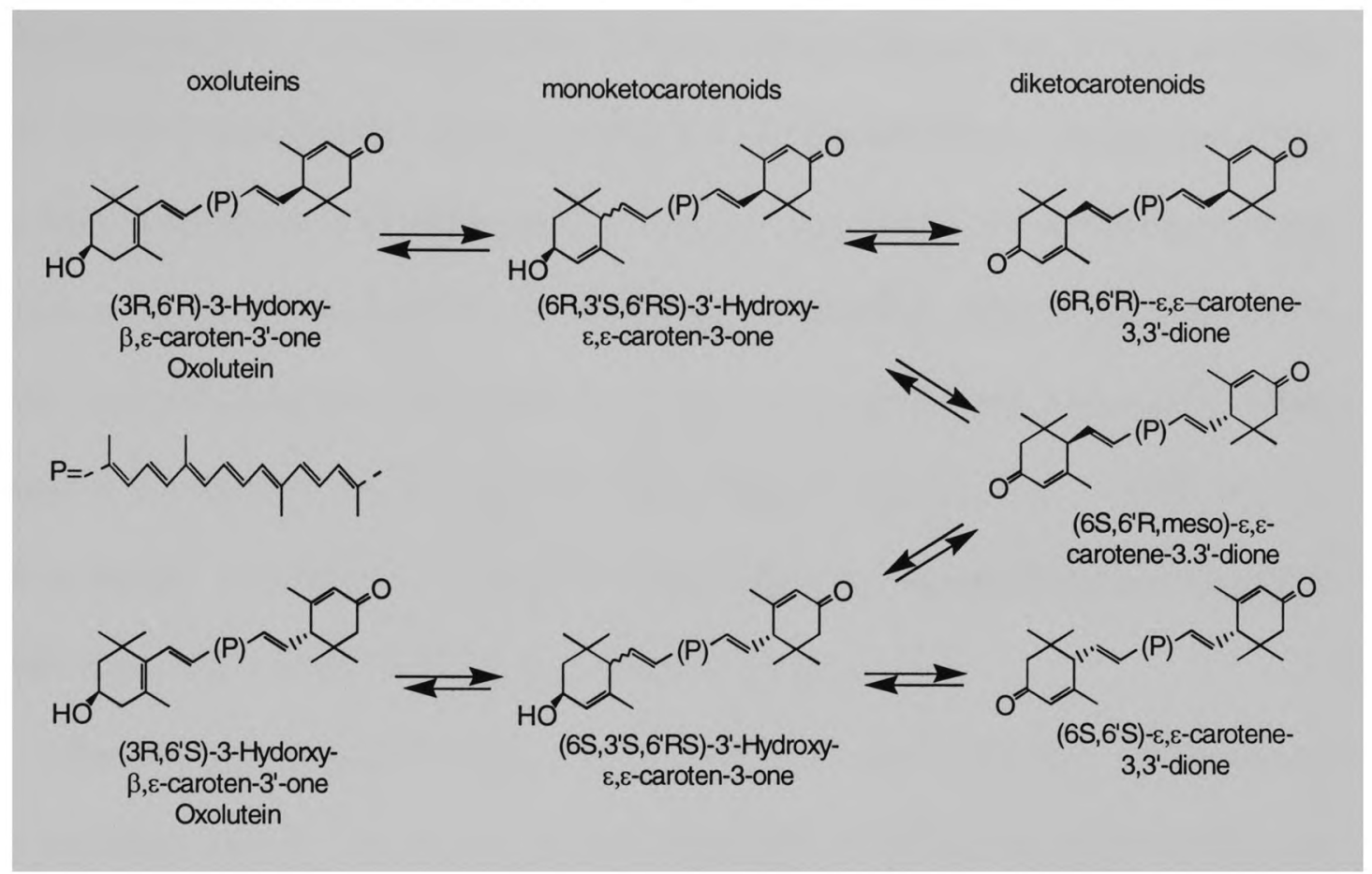

Figure 6. Formation of diketocarotenoids from oxoluteins (Khachik, et al. 1995)

In order to identify the possible oxidation products of the carotenoids, a powerful analytical technique, such as LC-MS is generally used. (Khachik et al., 1997) Although HPLC coupled with a UV detector cannot give exact structural information, it is still the most common method for carotenoid analysis due to it high sensitivity. This method has been used in our group for the analysis of carotenoids extracted from human serum. Figure 7 shows a typical chromatogram of the carotenoids found in human serum obtained using a reversed-phase HPLC with a UV detector. Several peaks can be identified with a high degree of confidence based upon their retention times. The most abundant peak, which elutes at $10.958 \mathrm{~min}$, is the lutein peak. Peaks at retention time $11.758,19.575,27.975,77.0$ and 84.90 min are identified as zeaxanthin, $\beta$-cryptoxanthin, 
$\alpha$-carotene and $\beta$-carotene, respectively. However, several peaks are found to be present for which the identity is not known. Two of them, referred to as A and B, are constantly present in the chromatograms of carotenoids extracted from human serum. These two peaks have been found to be lutein-related. They are suspected to be oxidation products of lutein in human serum based on the following observations. First, it has been found that the area of these two peaks increased with increases in the lutein peak which occurred in a long-term lutein supplementation study. (Landrum, et al., 1997) Second, based on reports in the literature (Khachik et al., 1995), the reported oxidation products of lutein should elute at earlier times than the hydroxycarotenoids.

The purpose of this part of this work is to further characterize these metabolites of lutein in human serum. The results of this study will be helpful in understanding the function of lutein as an antioxidant in human blood. The identification of the metabolites of lutein can also helpful in determination of the total concentration of lutein in serum.

\section{Experimental}

(1) Reagents and materials

Lutein and zeaxanthin were extracted from marigold flowers and guji berry, respectively. Lutein and zeaxanthin standards were prepared at a concentration ratio of 1:1. The concentrations of lutein and zeaxanthin were determined at wavelength $451 \mathrm{~nm}$ using an UV spectrometer. It is known that the molar absorption coefficient of lutein and zeaxanthin are 144800 and $140900 \mathrm{~L} /(\mathrm{mol}, \mathrm{cm})$ in ethanol.

HPLC grade of methanol, acetonitrile, triethylamine and hexane were purchased from Fisher. The reduction reagent sodium borohydride (98\%) was purchased from 
Aldrich Chemical Company. (Milwaukee WI 53233 USA) The nitrogen gas was purchased from Air Products and Chemical, Inc.

The blood samples were drawn from a healthy, female volunteer.

(2) Apparatus

Reversed-phase HPLC system (1) is a LDC/Milton Roy solvent delivery system equipped with a $50 \mu$ injection loop and it was coupled with a SM4000 UV-visible detector set at $451 \mathrm{~nm}$. The data was stored and processed by a Peak Simple computing system.

The reversed-phase HPLC system (2) is a LDC/Milton Roy solvent delivery system equipped with a $250 \mu$ injection loop and it was coupled with a Shimadzu SPDM6A photodiode array UV/Vis detector. The wavelength scan range is set at $195-600$ $\mathrm{nm}$. The data was stored and processed in a Shimadzu supplied software.

A Centrific Model 228 Centrifuge was used which was purchased from Fisher. A Fisher brand K-550-G Vortex was also used.

(3) Procedures

(i) Reduction of serum extract with $\mathrm{NaBH}_{4}$

Serum extraction: A pair of frozen serum samples (serum samples were obtained from a blood sample drawn from the same person on the same day) were chosen. They were left at room temperature for $30 \mathrm{~min}$ to allow them to defrost and then homogenized by shaking the sample vials gently several times to insure homogeneity of the sample. A 2 $\mathrm{ml}$ aliquot of serum was transferred into a $10 \mathrm{ml}$ centrifuge tube by a pipette. The serum proteins were precipitated by the addition of $2 \mathrm{ml}$ of ethanol/deionized water (50:50 $\mathrm{V}: \mathrm{V})$. The carotenoids were extracted with three $4 \mathrm{ml}$ portions of hexane, and followed 
by a $1 \mathrm{~min}$ vortex and $5 \mathrm{~min}$ centrifugation. The hexane layer was removed by pipette into a $2 \mathrm{ml}$ high-density polypropylene vial and evaporated to dryness under a gentle stream of nitrogen. One of the extracted carotenoid samples was treated with $\mathrm{NaBH}_{4}$ following the reduction reaction procedures. Another extract was analyzed directly without treatment with $\mathrm{NaBH}_{4}$ for the purpose of the comparison. All the procedures were preformed carefully in a dim light condition.

Reduction reaction: About $1 \mathrm{~g} \mathrm{NaBH}_{4}$ in $10 \mathrm{ml} \mathrm{MeOH}$ solution was prepared in a round bottom flask. $100 \mu \mathrm{l}$ of this solution was transferred to a high-density polypropylene vial that contained the extracted carotenoids. The reduction reaction occurs rapidly and after a 10 min period, $1 \mathrm{ml}$ of deionized water was added to decompose the excess $\mathrm{NaBH}_{4}$. The solution obtained was carefully transferred into a $10 \mathrm{ml}$ centrifuge tube with pipette. The reduced carotenoids were extracted with three portions of $3 \mathrm{ml}$ hexane and followed by 1 min vortex and 5 min centrifugation. The hexane layer was removed and dried under a stream of nitrogen.

HPLC analysis: Carotenoids extracted from serum with or without reduction by $\mathrm{NaBH}_{4}$ were analyzed by the reversed-phase HPLC system (1) equipped with an SM4000 UVvisible detector set at $451 \mathrm{~nm}$. The data was stored and processed using the Peak Simple data acquisition system. The column used was a $250 \times 4.6 \mathrm{~mm}$ Ultracarb C18 $3 \mu \mathrm{m}$ ODS (Phenomenex ${ }^{\mathrm{TM}}$ ) which was protected with a $\mathrm{C} 18$ Phenomenex ${ }^{\mathrm{TM}}$ guard cartridge. The mobile phase consisted of an isocratic mixture of acetonitrile $85 \%, \mathrm{MeOH} 15 \%$ with $1 \mathrm{ml}$ triethylamine per liter of mobile phase added to inhibit degradation of the carotenoids on column. The flow rate was $1.0 \mathrm{ml} / \mathrm{min}$. The injection volume was $20 \mu \mathrm{l}$. The chromatograms of pre- and post- reduction reaction were shown in Figure 7 and Figure 8. 
(ii) Reduction of separated compounds $\mathrm{A}$ and $\mathrm{B}$ with $\mathrm{NaBH}_{4}$

Carotenoids were extracted from $2 \mathrm{ml}$ serum according to the procedure described above. The carotenoid extracts were analyzed using the reversed phase HPLC system (1). The separated compounds A and B were carefully collected into two $2 \mathrm{ml}$ vials. After a number of injections, the collected compounds A and B were dried under a stream of nitrogen gas. Since the retention times of compounds A and B are very close, further purification was carried out by adding $20 \mu \mathrm{l}$ of ethanol to the dried samples which were re-separated using the same HPLC system. Chromatograms obtained for the re-injection analysis of compounds A and B are shown in Figure 9 and 10, respectively. It can be seen that there is a small shoulder (Figure 9) resulting from a trace of compound B in the purified sample of compound A. Similarly, the small peak (Figure 10) eluting prior to compound $\mathrm{B}$ is a trace of compound $\mathrm{A}$ and the small shoulder with a longer elution time is lutein. Compounds $\mathrm{A}$ and $\mathrm{B}$ were separately collected into $2 \mathrm{ml}$ vials, then dried with a stream of nitrogen gas. Reduction reactions were carried out for both compound A and B following the procedure described above. After reduction, both samples were analyzed by reversed-phase HPLC system as above and the products of the reactions were collected and dried. Typical chromatograms obtained for the reduced A and B are shown in Figure 11 and 12 . These reduced compounds were also co-injected with the lutein/zeaxanthin (1:1) standard and the results are shown in Figure 13, 14 and 15.

(iii) Comparisons of UV absorption Spectrum

The extracted carotenoids from $2 \mathrm{ml}$ serum were analyzed by the reversed-phase HPLC system (2) with a $250 \mathrm{ml}$ injection loop and followed by a Shimadzu SPD-M6A photodiode array UV/Vis detector. In order to obtain a good quality spectrum, a $250 \times 10.0$ 
$\mathrm{mm}$ Ultracarb $3 \mu \mathrm{m}$ ODS column was used. The carotenoids extracted from $2 \mathrm{ml}$ serum can be totally introduced to the column in one injection using this setup. The flow rate was $4.0 \mathrm{ml} / \mathrm{min}$. and a typical chromatogram for the carotenoid analysis is shown in Figure 16. Compounds A and B are well separated and were collected separately. The UV absorption spectrum of lutein, compound A and B were obtained and are shown as in Figures17, 18, and 19. The collected compounds A and B were subjected to reduction following the previously described procedures. After reaction, the products were analyzed using the same HPLC/UV/Vis system. The absorption spectra were recorded over the wavelength range of $195-600 \mathrm{~nm}$.

\section{Results and Discussion}

As mentioned before, peaks A and B were two unknown components. They eluted before lutein on a reversed-phase HPLC. (Figure 7) Both compounds A and B were thought to be the oxidative metabolites of the hydroxy-carotenoids. One or two carbonyl groups may be present on the ionene end-rings. Lutein is the most abundant hydroxycarotenoid found in human blood. It has been suggested that lutein functions as an antioxidant. If this is true, mono- or di-keto forms of lutein are expected to be found in serum. Figures 5 and 6 show several of the possible mono- or di-keto oxidative metabolites of lutein. The suspected carbonyl on the compounds A and B, if present, should be reduced by $\mathrm{NaBH}_{4}$ to produce a hydroxyl group. The structures of reduced $\mathrm{A}$ and B might be expected to be that of lutein or lutein stereoisomers. In this study, three experiments were designed to test this hypothesis.

In the experiment Reduction of serum extract with $\mathrm{NaBH}_{4}$, the serum extract was directly reduced with $\mathrm{NaBH}_{4}$ and the products were reanalyzed and compared with 
the serum extract obtained prior to reduction. It was found that the peak height of compounds A and B decreased significantly after the reduction reaction. (Figure 8) However, no significant increase in the peak area of lutein can be observed because of the low concentration of $\mathrm{A}$ and $\mathrm{B}$ in comparison to the concentration of lutein in the extract. The changes in peak area ratios, which result from reduction of $\mathrm{A}$ and $\mathrm{B}$, are shown in Table. 1. Lutein is stable toward the treatment by $\mathrm{NaBH}_{4}$. The increases of peak ratios for both $\mathrm{A}$ and $\mathrm{B}$ indicate that both compounds react with $\mathrm{NaBH}_{4}$. The result of this experiment is consistent with the presence of a carbonyl group in both compounds $\mathrm{A}$ and B.

\begin{tabular}{lccc}
\hline & Before Reduction & After Reduction & Changes in Ratio \\
\hline Lutein peak area/Peak A area & 10.30 & 54.17 & 43.87 \\
Lutein peak area/Peak B area & 12.01 & 97.05 & 85.04 \\
\hline
\end{tabular}

Table 1. Changes in peak ratio of Lutein peak /A and Lutein peak /B

In order to further characterize these two unknown compounds, Reduction of the separated compounds A and B with $\mathrm{NaBH}_{4}$ was conducted. Because the retention times of lutein and these two compounds are very close, separation and collection was done twice in order to obtain pure samples. (Figures 9 and 10) Reduction reactions were performed for both separated A and B. For compound A, two peaks, $A_{1}$ and $A_{2}$, were observed on the HPLC chromatogram after reduction at retention times of 9.116 and 10.366 minutes, respectively. (Figure 11) An additional peak of variable height was 
observed at about 19 min. Since peak $A_{1}$ has the same retention time as Peak A, its identity was consistent with some unreacted compound A. Peak $\mathrm{A}_{2}$ was collected and coinjected with a lutein/zeaxanthin standard (1:1). There was not an observable increase in the peak area of the lutein peak. (Figure 14) However, a small peak was observed at retention times of $10.483 \mathrm{~min}$, which is the time of $\mathrm{B}$ elution in the chromatogram, where no peak can be found in the chromatogram of the lutein/zeaxanthin (1:1) standard. (Figure 13)

There are three peaks labeled $B_{1}, B_{2}, B_{3}$ and an unknown peak that can be observed in the chromatogram obtained by reduction of product $\mathrm{B}$ at retention times of $9.725,10.316,11.075$ and $20.658 \mathrm{~min}$, respectively (Figure 12). $\mathrm{B}_{1}$ was considered as the impurity of compound $A$ and $B_{2}$ was unreacted compound $B$. Peak $B_{3}$ has same retention time as lutein, peak $B_{3}$ was collected and co-injected to the lutein/zeaxanthin (1:1) standard and a significant increase in peak high of the lutein peak was observed. (Figure 15) These results are consistent with the identity of component $B$ as an oxidative product of lutein possessing a carbonyl group. After reduction reaction, B was in part reduced to form lutein.

In order to prove the result obtained from the above experiment, comparisons of UV absorption spectra were performed. Figure 16 to Figure 27 show the results. The spectrum of peak $B_{3}$ is identical to the UV absorption spectrum of an authentic lutein standard. (Figure 17 and Figure 22) Spectra of A and B are shown in Figure 18 and Figure 19. They are lutein-like, but the $\lambda_{\max }$ of $A$ is $5 \mathrm{~nm}$ shorter than that of lutein. The keto groups in the oxidative products of lutein are expected to be on $\mathrm{C} 3$ ' of the $\varepsilon$-end ring. These carbonyls are not expected to be conjugated with the extended polyene 
system. Therefore, reduction of these keto groups has little or no effect on the absorption spectra of the compounds. In other words, they should have similar spectra to lutein. Coinjection was also performed in this part of the experiment. The results agree with those obtained above. Peak $B_{3}$ caused a significant increase in lutein peak. (Figure 25) The same result was obtained from the co-injection of reduced compound A with the lutein/zeaxanthin (1:1) standard. (Figure 23) There is only a marginal increase in peak area observed, (Figure 24) but this may be only because the amount of material is small.

An unexpected result was obtained in this study. An unknown peak was found in both chromatograms of reduced compounds A and B. (Figure 11, 12, 20 and 21) The retention time of this unknown is about $19-20$ min which is near the time of $\beta$ cryptoxanthin elution in the chromatogram of serum analysis. (Figure 7) A side reaction might occur during the reduction process. The spectrum of this unknown peak from both reduced compounds A and B was also obtained and is shown in Figures 26 and 27. These spectra are both similar. Since an authentic standard of $\beta$-cryptoxanthin is not available, the further comparison was not pursued. It appears to be a single compound produced from both $\mathrm{A}$ and $\mathrm{B}$.

\section{Conclusion}

The UV absorption spectra of authentic lutein, compound A and B are shown in Figure 17, 18 and 19. The UV absorption spectrum of compound B is identical as that of authentic lutein. Compound A has unique spectroscopic with $\lambda_{\max } 5 \mathrm{~nm}$ shorter than that of the lutein. The two unknown compounds $\mathrm{A}$ and $\mathrm{B}$ both react with $\mathrm{NaBH}_{4}$. They appear to be oxidized carotenoids containing one or more carbonyl function groups. B is reduced 
to form lutein. Compound B might be either the mono- or diketo- form of lutein. Characterization of compound A has proven difficult.

A comparison experiment, oxidization of lutein, would be very helpful for further identification and this project is currently in progress. The structures of lutein oxidation products can be identified by NMR since sufficient quantities can be readily prepared. LC-MS will also be essential for final identification of these compounds. This technique was not available during this study but is also currently under way. 


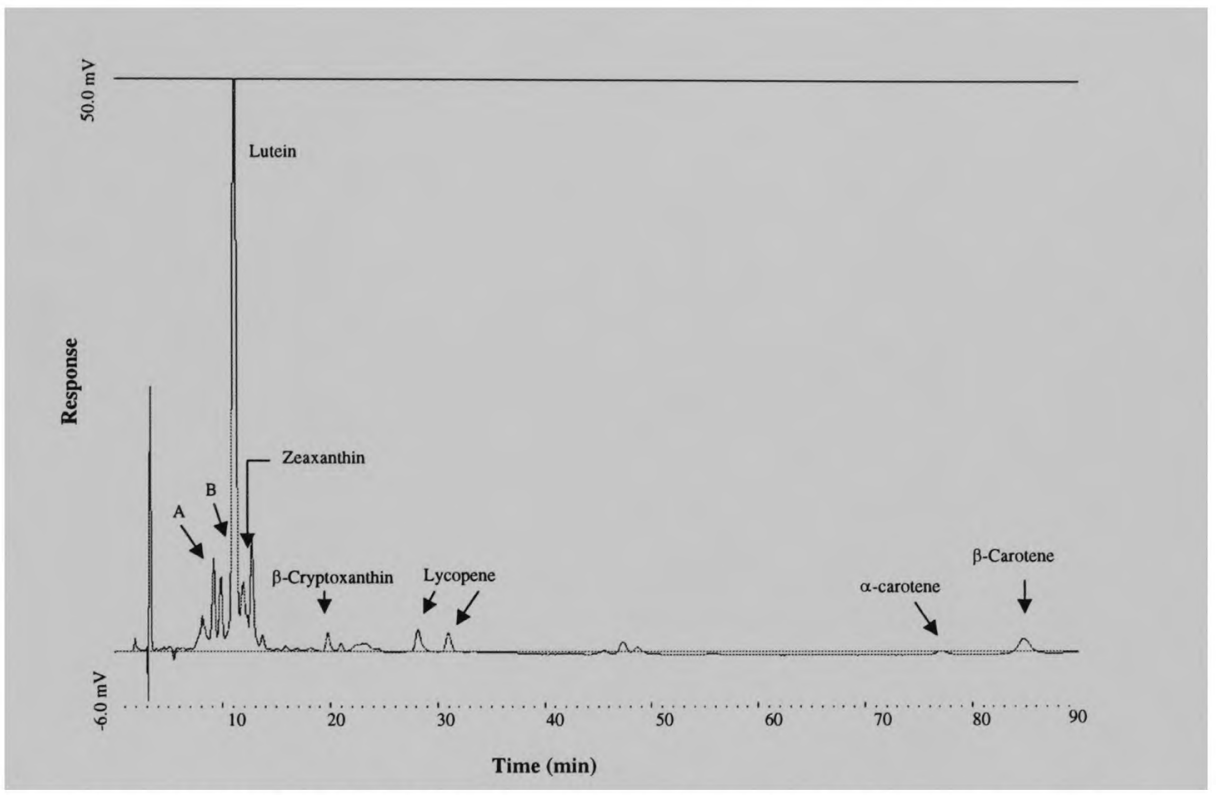

Figure 7. Typical HPLC chromatogram of carotenoids from serum extract

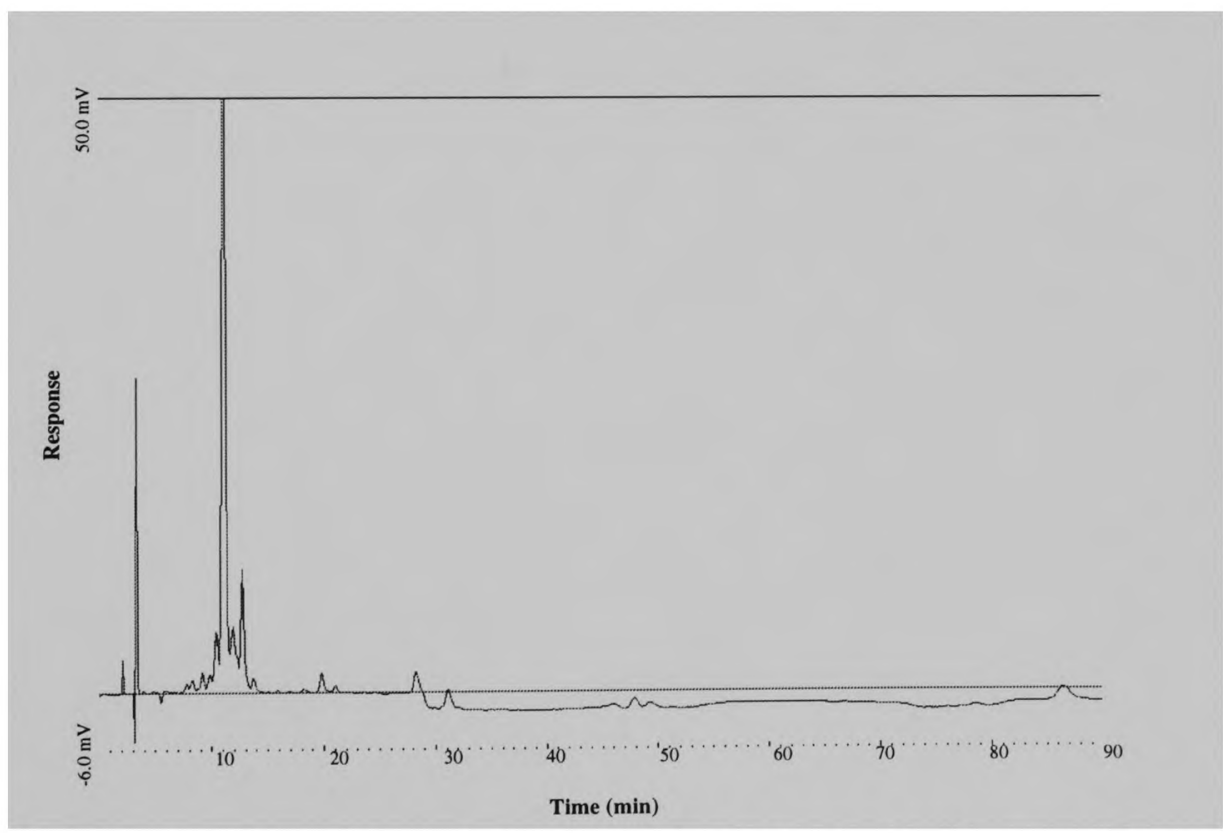

Figure 8. HPLC chromatogram of carotenoids from reduced serum extract 


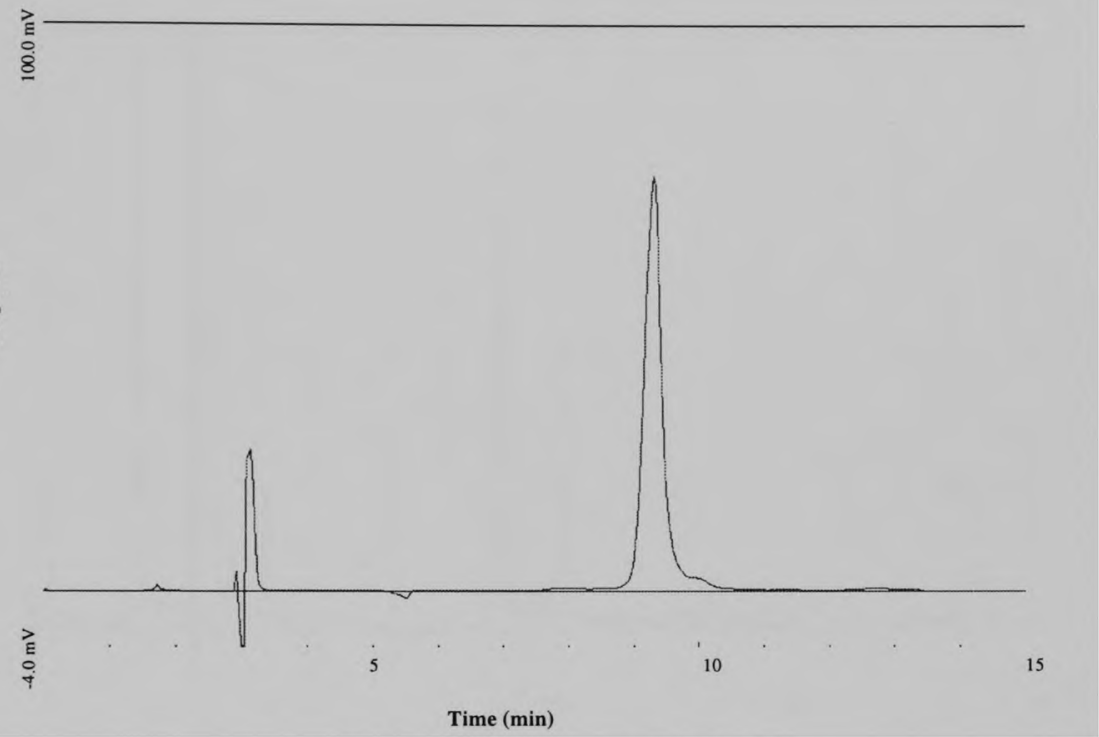

Figure 9. Purified lutein metabolite A

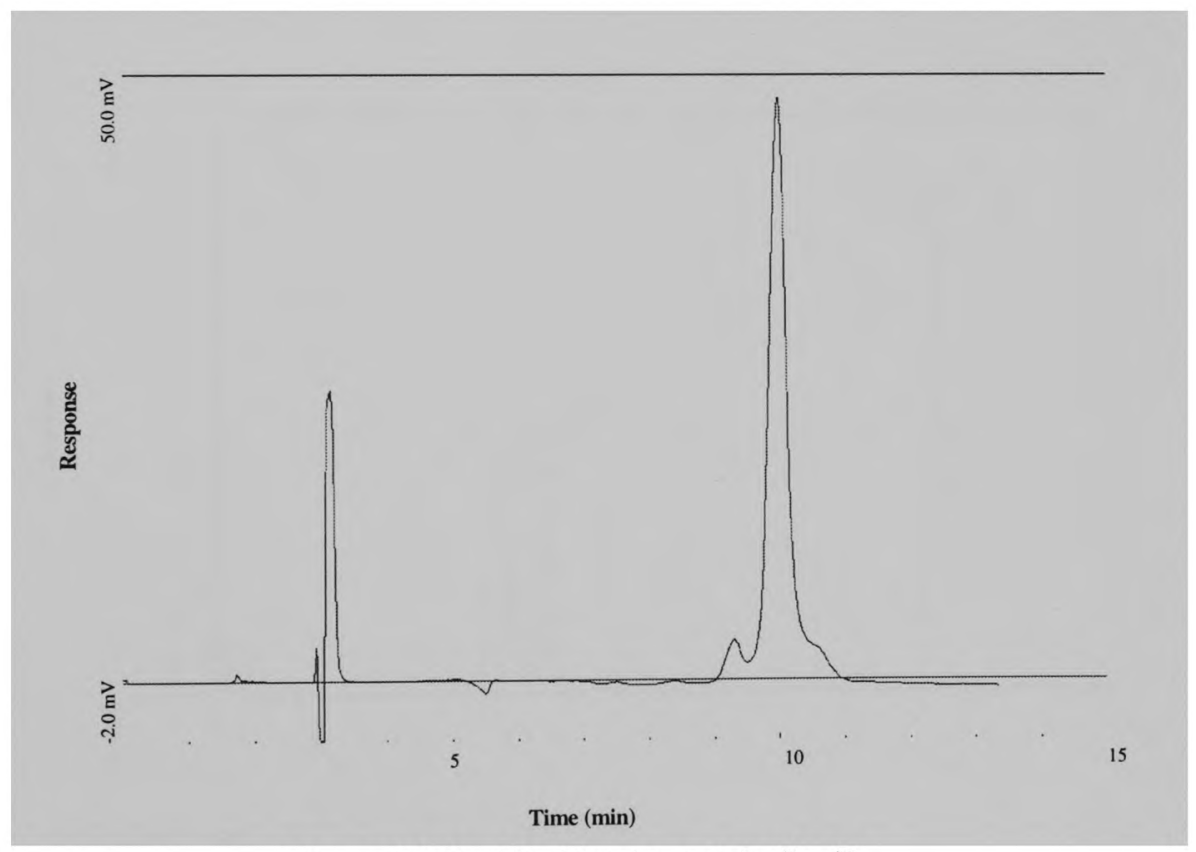

Figure 10. Purified lutein metabolite B 


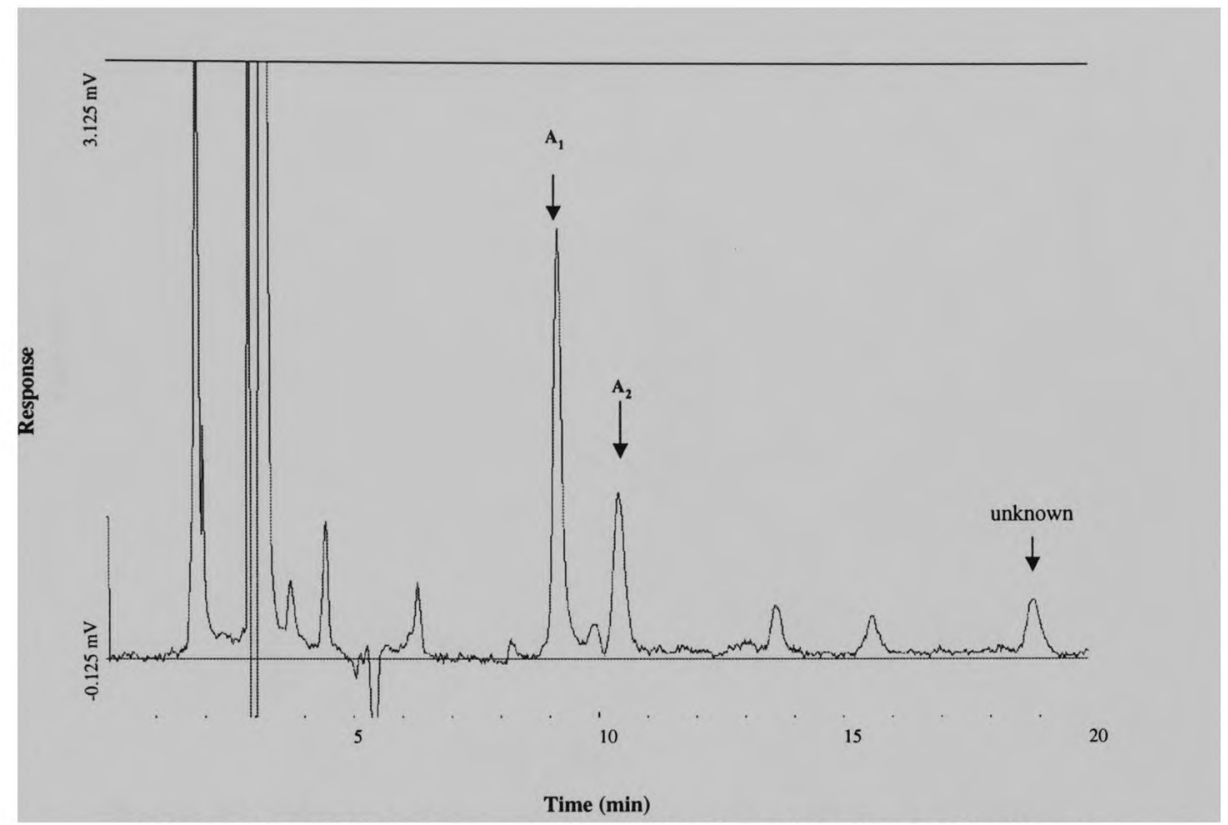

Figure 11. HPLC chromatogram of reduced lutein metabolite A

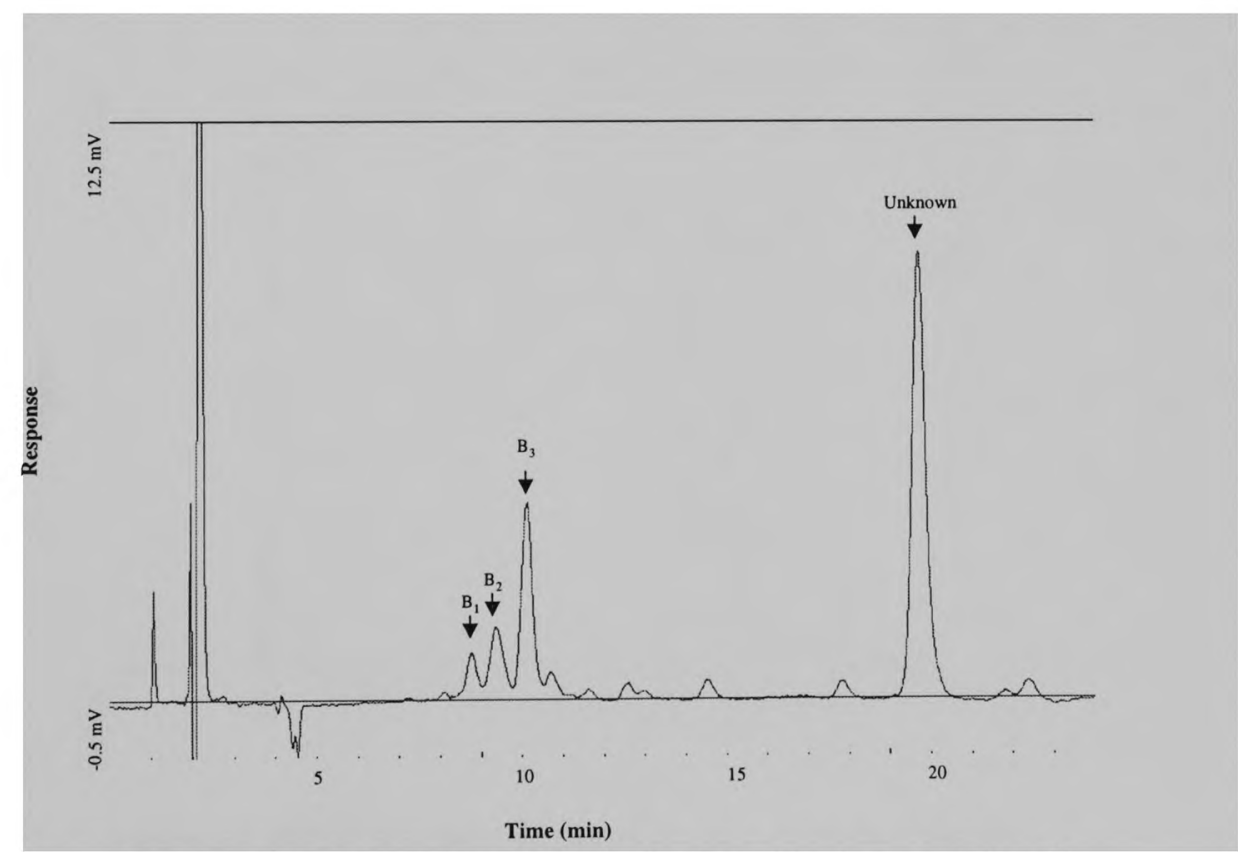

Figure 12. HPLC chromatogram of reduced lutein metabolite B 


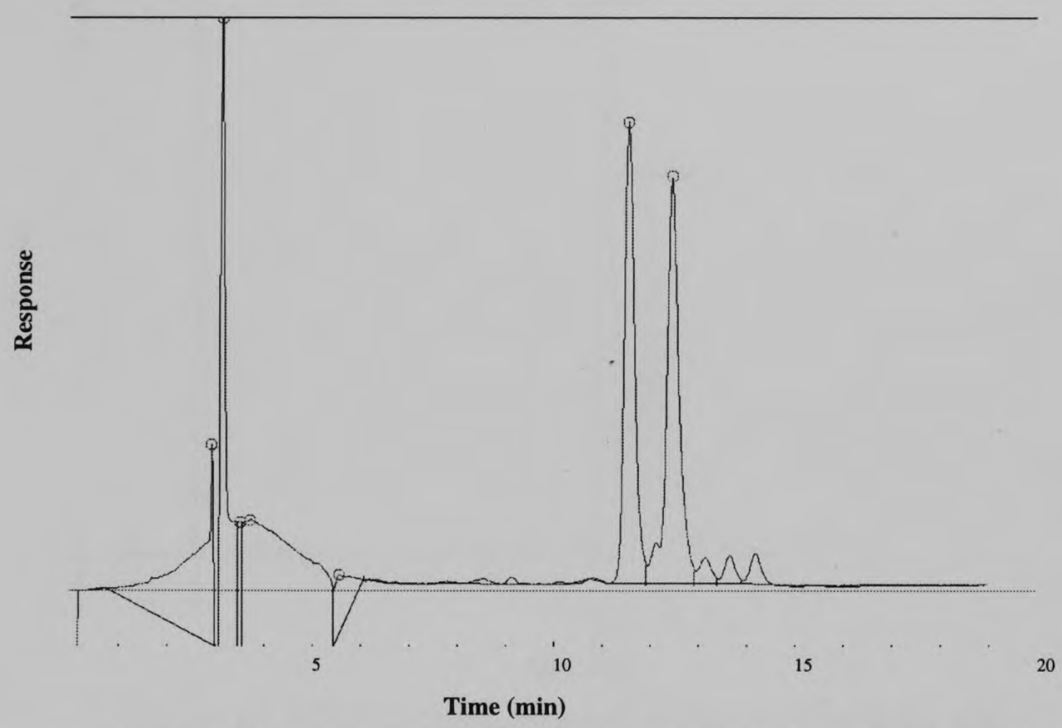

Figure 13. HPLC chromatogram of lutein/zeaxanthin (1:1) standard

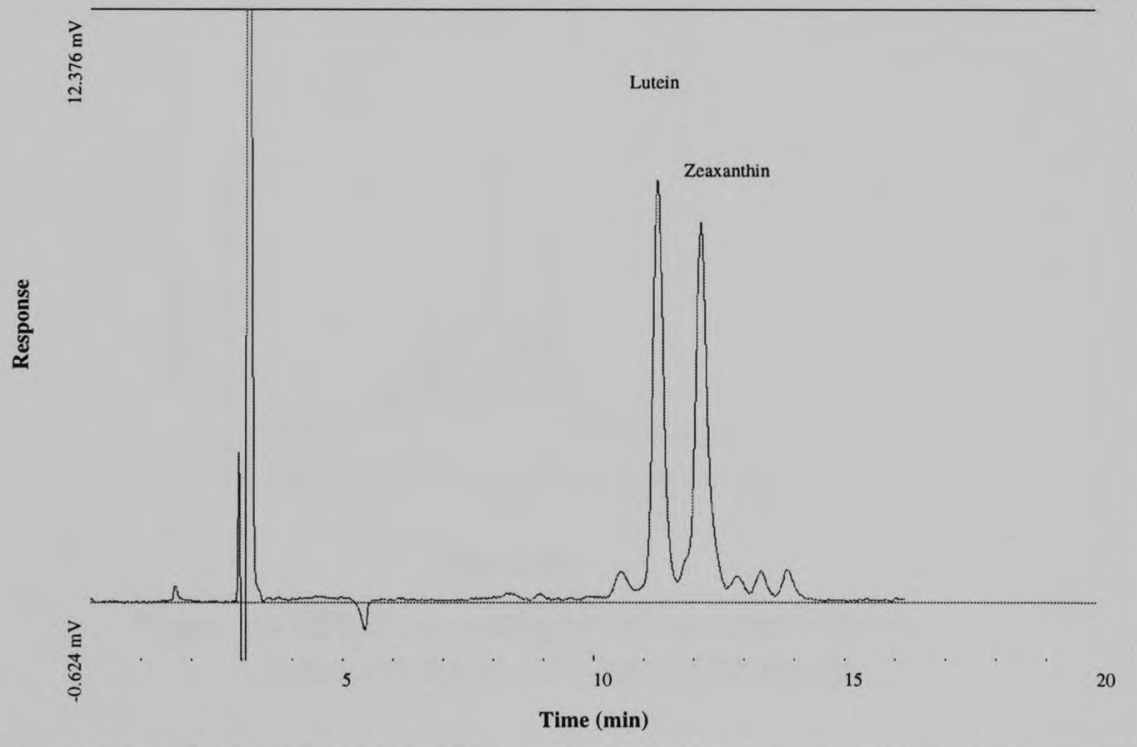

Figure 14. HPLC chromatogram of co-injection peak $A_{2}$ with lutein/zeaxanthin (1:1) standard 


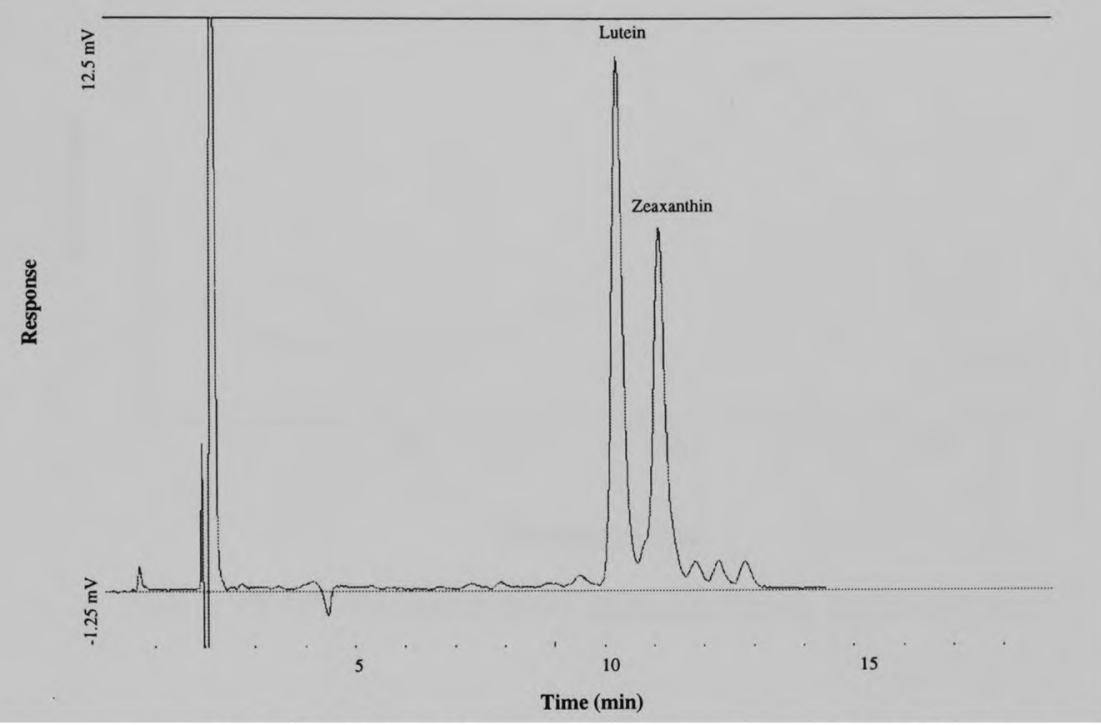

Figure 15. HPLC chromatogram of co-injection peak $B_{3}$ with lutein/zeaxanthin (1:1) standard

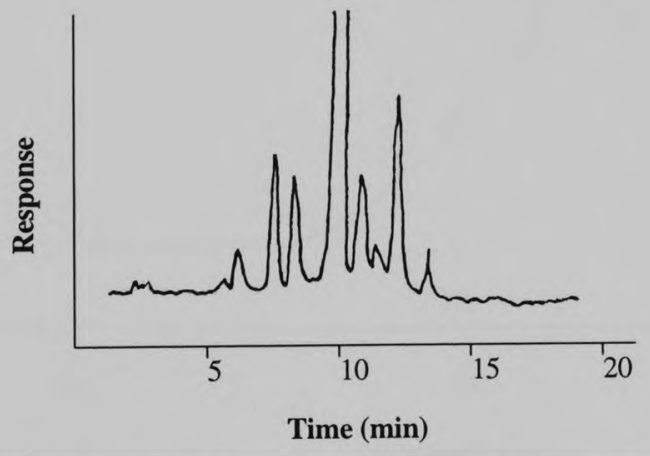

Figure 16. HPLC chromatogram of carotenoids from serum extract on a $10.00 \mathrm{~mm} \mathrm{C18} \mathrm{column}$ 


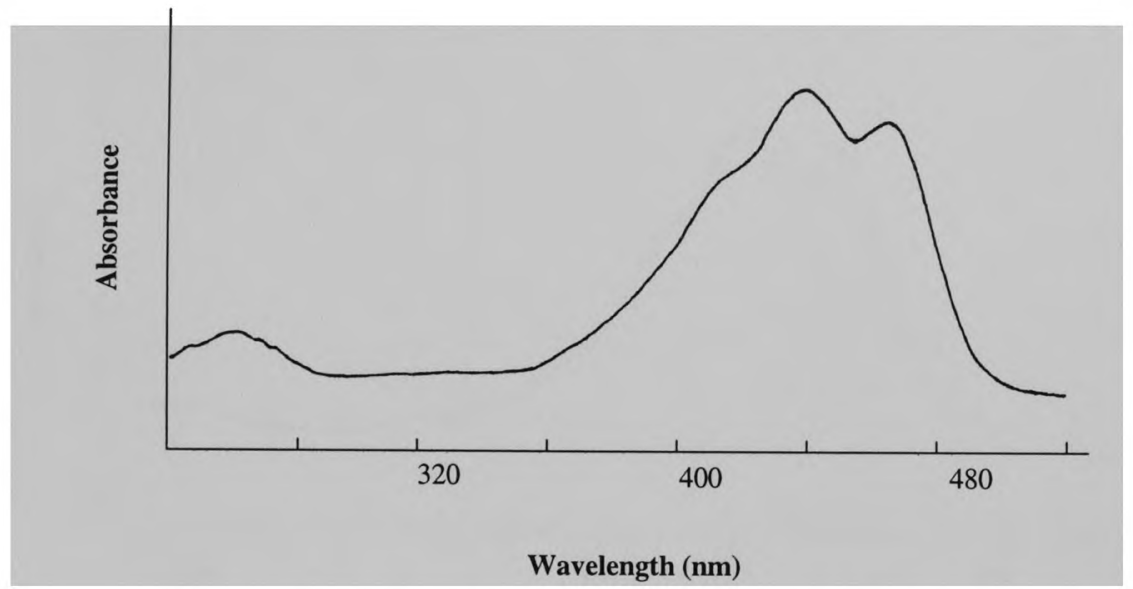

Figure 17. UV/Vis spectrum of authentic lutein, $\lambda \max =446 \mathrm{~nm}$

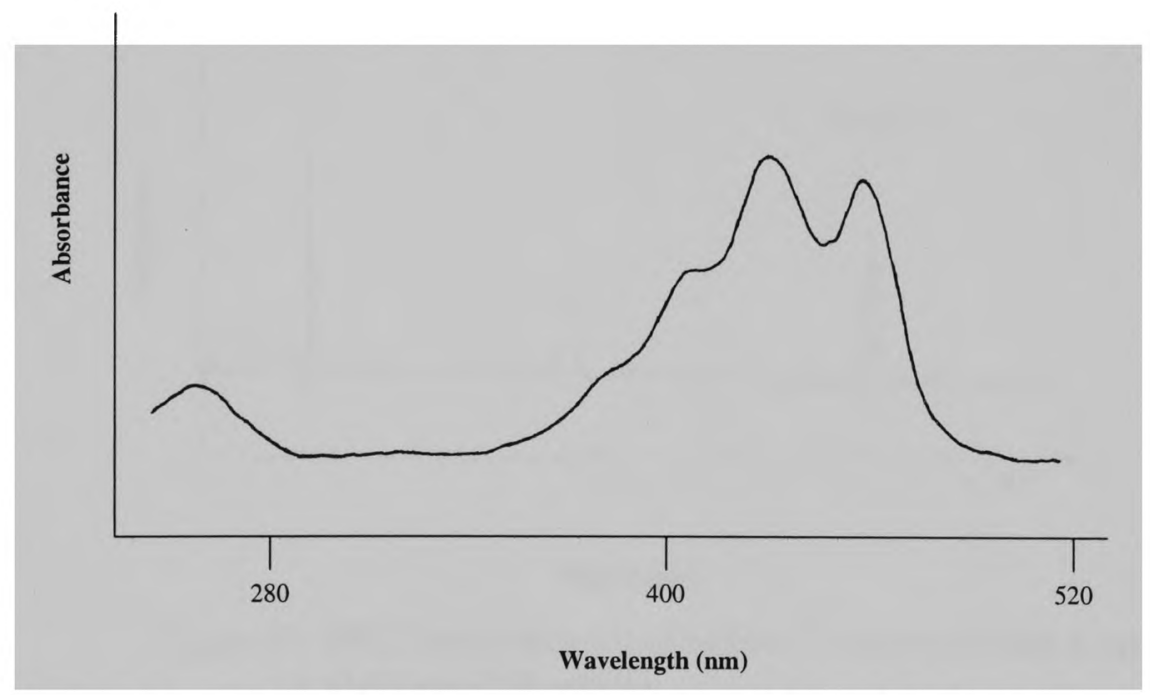

Figure 18 . UV/Vis spectrum of lutein metabolite $A, \lambda \max =441 \mathrm{~nm}$ 


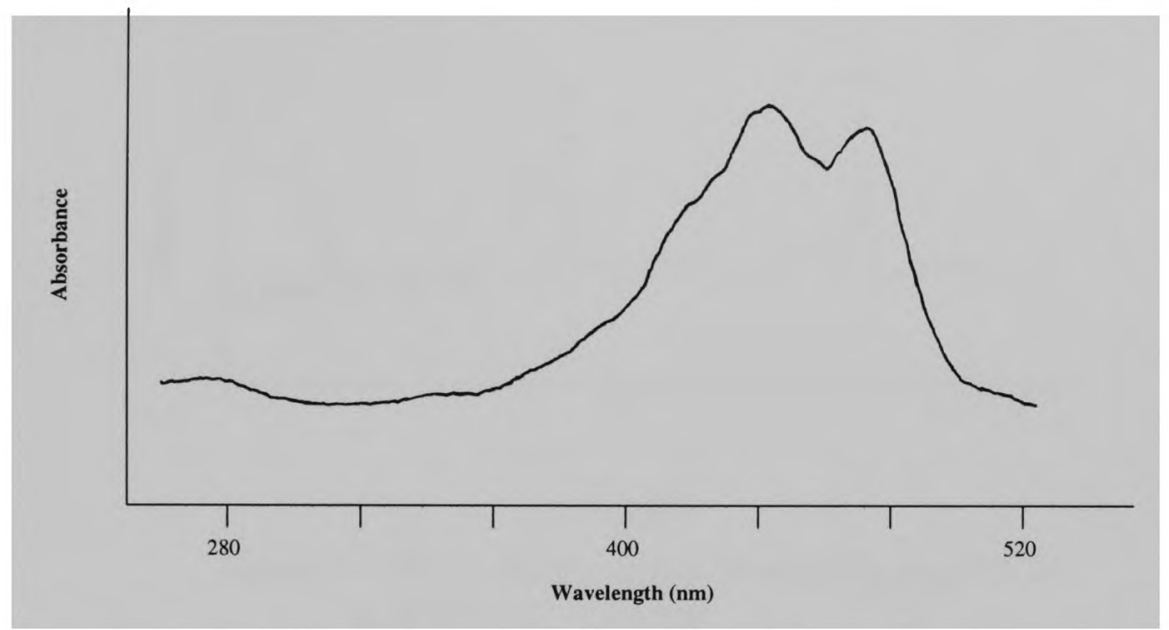

Figure 19. UV/Vis Spectrum of lutein metabolite $B, \lambda \max =446 \mathrm{~nm}$

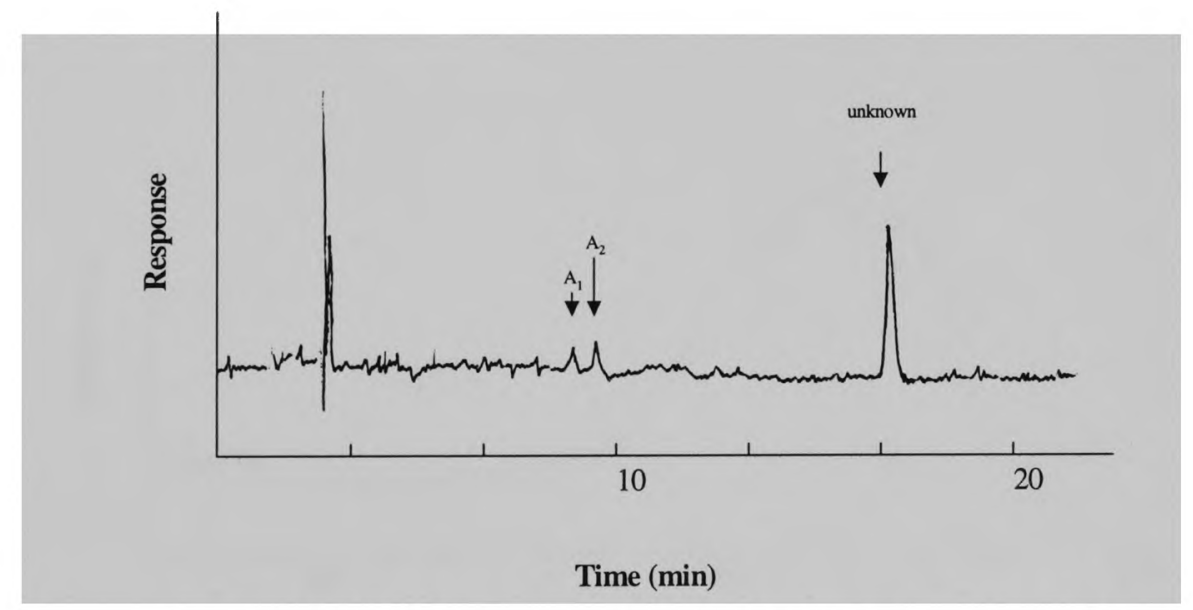

Figure 20. HPLC chromatogram of reduced lutein metabolite A on a $10.00 \mathrm{~mm} \mathrm{C18} \mathrm{column}$ 


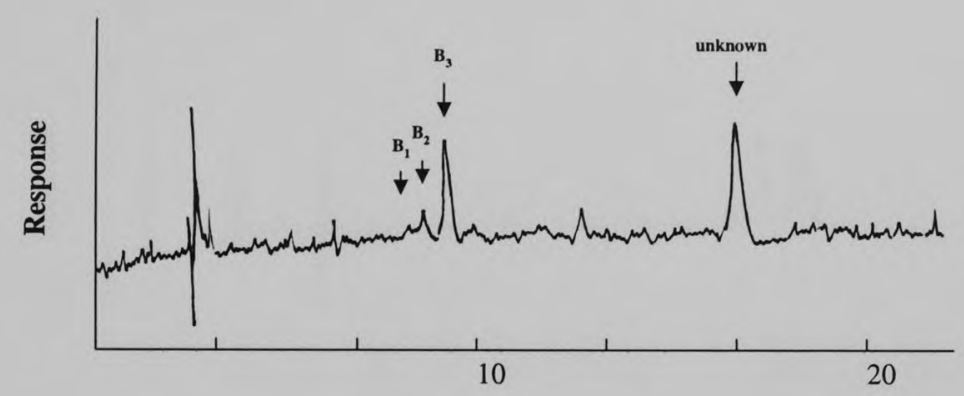

Time (min)

Figure 21. HPLC chromatogram of lutein metabolite B on a $10.00 \mathrm{~mm} \mathrm{C18}$ column

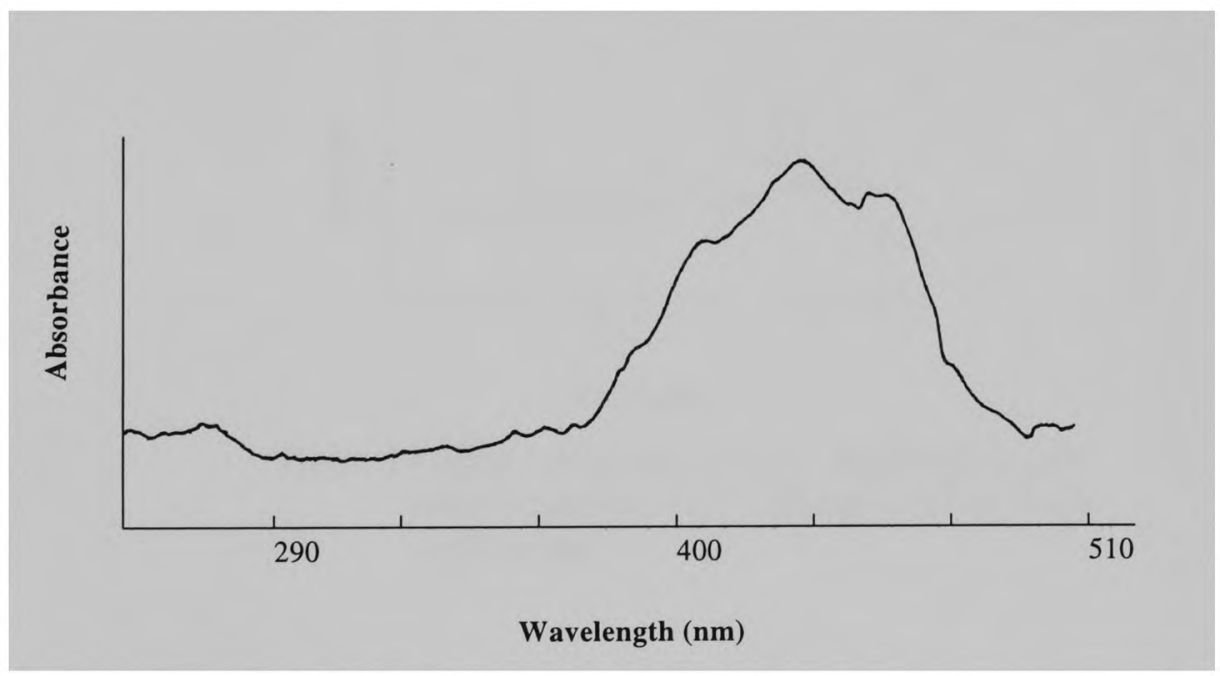

Figure 22. UV/Vis spectrum of $B_{3}, \lambda \max =450 \mathrm{~nm}$ 


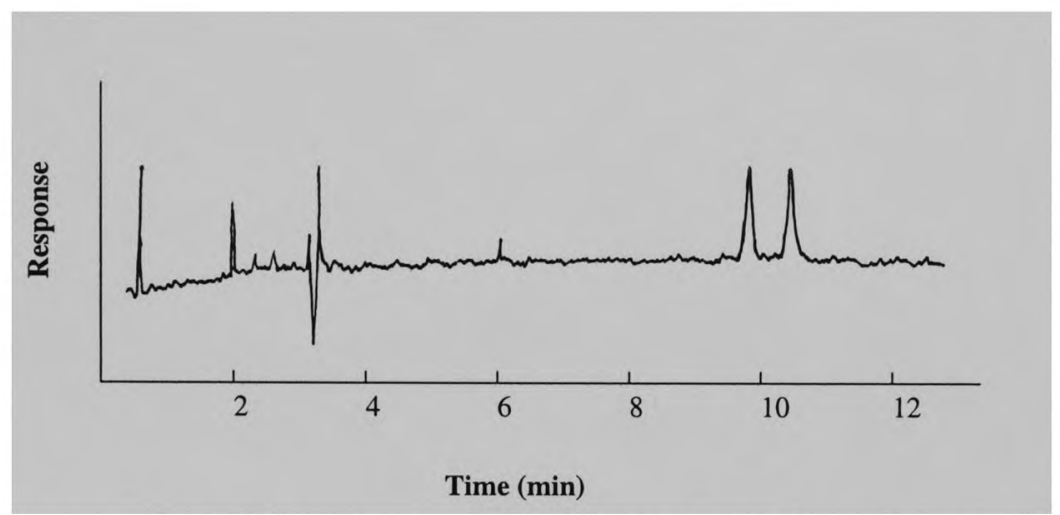

Figure 23. HPLC chromatogram of lutein/zeaxanthin (1:1) standard on a $10.00 \mathrm{~mm}$ C18 column

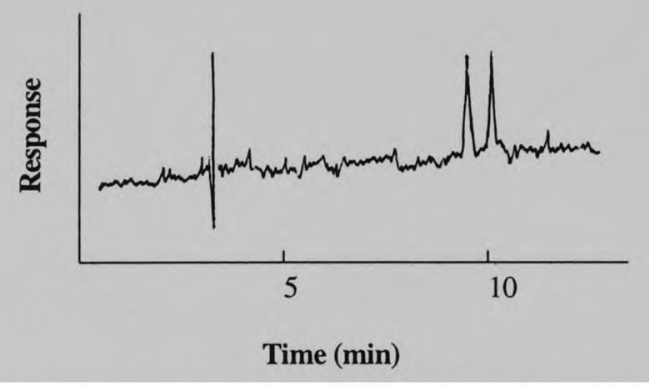

Figure 24. HPLC chromatogram of co-injection $\mathrm{A}_{2}$ with lutein/zeaxanthin (1:1) standard on a $10.00 \mathrm{~mm}$ C18 column 


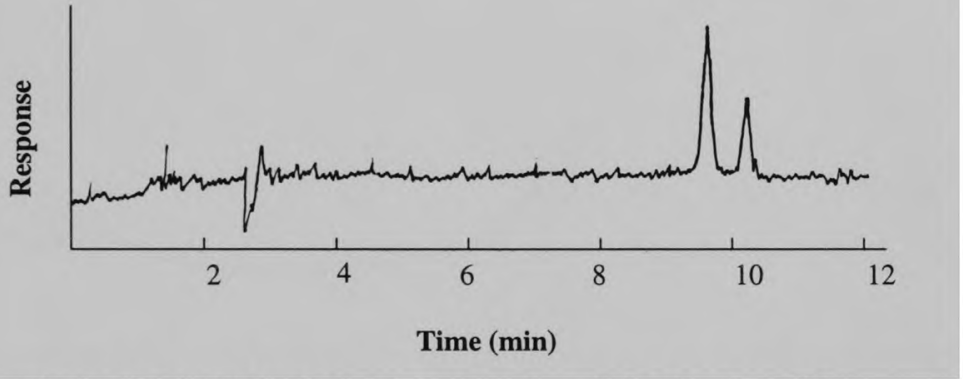

Figure 25. HPLC chromatogram of co-injection $\mathrm{B}_{3}$ with lutein/zeaxanthin (1:1) standard on a $10.00 \mathrm{~mm}$ C18 column

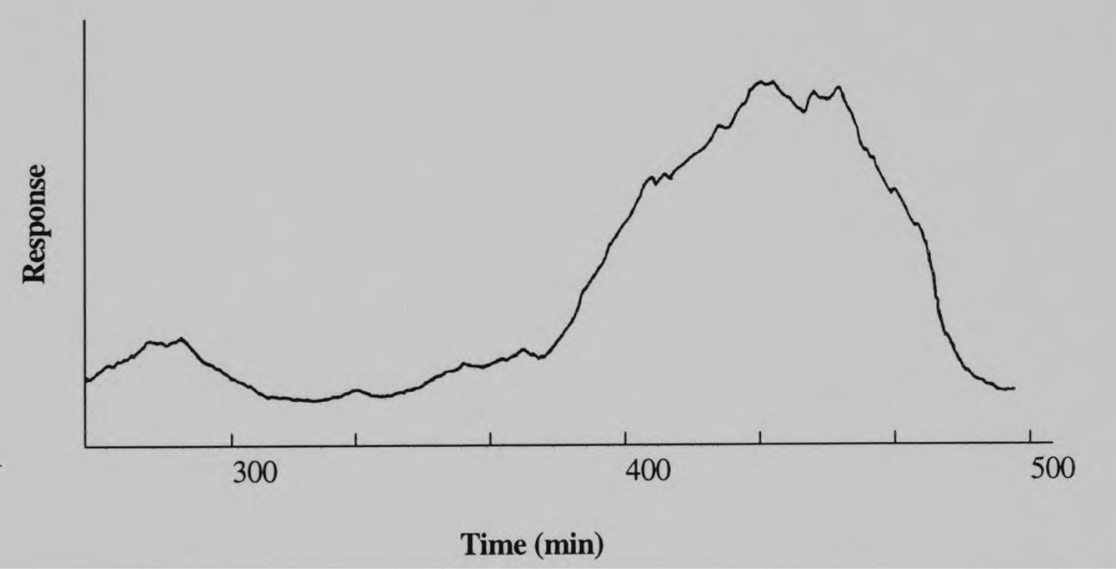

Figure 26. UV/Vis spectrum of unknown peak from the chromatogram of reduced lutein metabolite $A, \lambda \max =452 \mathrm{~nm}$ 


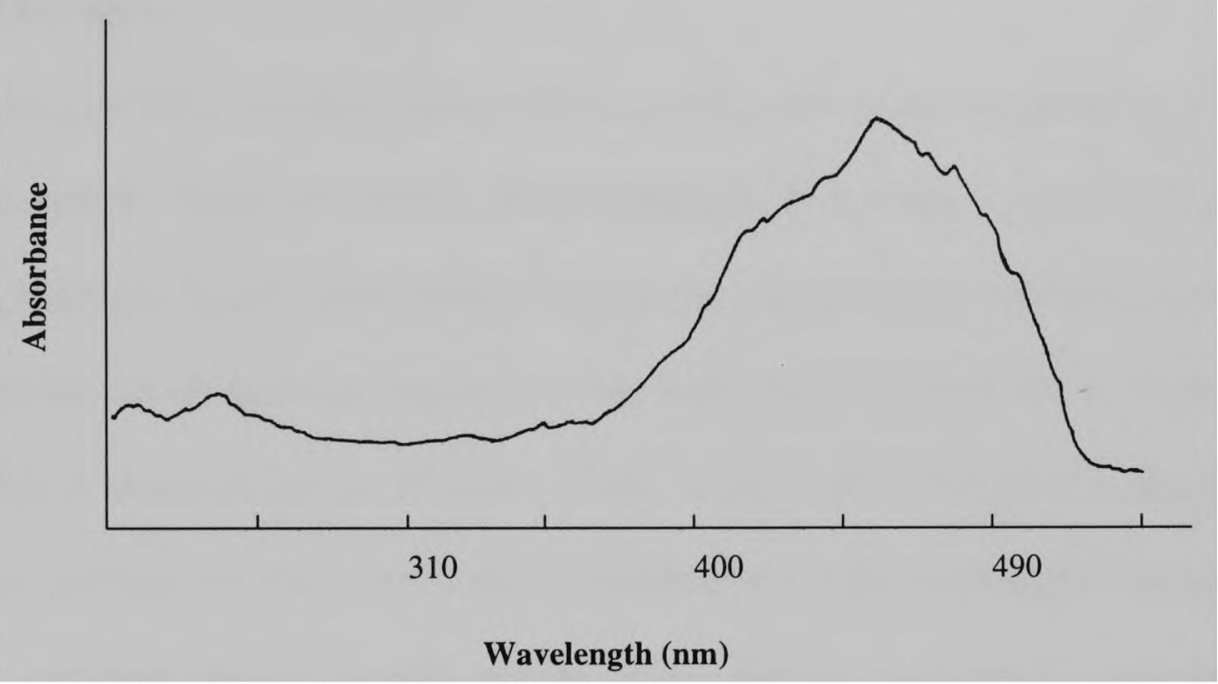

Figure 27. UV/Vis spectrum of unknown peak from the chromatogram of reduced lutein metabolite $B, \lambda \max =456 \mathrm{~nm}$ 


\section{PART 2 ZEAXANTHIN SUPPLEMENTATION STUDY}

\section{Overview}

Zeaxanthin is a yellow pigment that is widespread in nature. It occurs abundantly in maize, certain algae and bacteria. Zeaxanthin is $\beta, \beta$-carotene-3, 3'-diol (Figure 1). It is found in human serum. Although it is not the most abundant carotenoid in serum, it is a main component of macular pigment in the inner macula where the concentration of zeaxanthin is about twice that of lutein. (Bone, et al., 1997) The macular pigment has a protective function for the central retina. It has been reported that a significant increase in macular pigment optical density occurs when dietary supplements increase serum xanthophyll levels. (Landrum, et al., 1997) In this study, we have found the relationship of the serum response to dietary supplementation of zeaxanthin.

Among the number of methods that have been employed to investigate the absorption of carotenoids in humans, the most common one is the chronic dosing of a carotenoid and the measurement of the blood response. (Bowen, et al., 1993) The carotenoid may be in the form of a purified compound or ingested as a food. Healthy human subjects are given quantitated amounts of carotenoids, and changes in serum concentrations at various time intervals are determined. This method does not provide the absolute quantity or percent of the carotenoid absorbed. Some other methods of choice are metabolic balance techniques using isotopically labeled carotenoids. The metabolic balance technique involves the estimation of total carotenoid intake for a period of days called the balance period and the collection and analysis of all fecal output for carotenoids for the same time period. Output is subtracted from input and divided by the 
number of days of the balance period to obtain the quantity of carotenoid absorbed in the body per day. These methods are complicated. They do provide more definitive data for the characterization and quantitation of carotenoid absorption in human. The method of chronic dosing and measurement of serum response has been employed in this experiment. The concentrations of carotenoids in the serum were measured using reversed-phase HPLC.

\section{Experimental}

(1) Reagents and materials

HPLC grade Methanol, Acetonitrile, Hexane and Ethanol were purchased from Fisher. Pure lutein was obtained from Kemin Foods, Inc.

(2) Apparatus

Reversed-phase HPLC system (1) is a Waters 2690 separation module coupled with a Waters 486 tunable absorbance UV/Vis detector.

Reversed-phase HPLC system (2) is an LDC Analytical Consta Metric 3200 solvent delivery system equipped with a $250 \mu$ injection loop and coupled with an LDC/Milton Roy UV detector.

(3) Procedures

One healthy, male, non-smoker volunteer supplemented his diet with $30 \mathrm{mg}$ of zeaxanthin per day for 120 days. Blood samples were drawn every two days in the beginning of the supplementation period and also at the end of that period. At other times, blood samples were drawn weekly. The concentration of zeaxanthin in the serum before supplementation was also measured as a baseline level. All serum samples were analyzed by reversed-phase HPLC. An internal standard, mono-hexyl lutein (MHL), was 
used to quantify carotenoids. The concentrations of zeaxanthin and other carotenoids were determined from the peak area of the internal standard since the quantity of the internal standard is known. It has been previously shown that both the internal standard and lutein have the same absorption coefficient. (Sprague, 1997) The relative differences in absorption were determined by comparing the serum carotenoids concentration at various post-ingestion intervals with the baseline values.

\section{(i) Sample preparation}

Blood was drawn into a $5 \mathrm{ml}$ Vacutainer serum-separator tube by venipuncture. After allowing at least $30 \mathrm{~min}$ for coagulation, the blood samples were centrifuged for 10 min and the serum portion was removed by a disposable pipette and placed into highdensity polypropylene $2 \mathrm{ml}$ vials which are fitted with a rubber O-ring seal. Serum was filled to the capacity to minimize any potential oxidation by air. Samples were analyzed immediately or stored at $-20^{\circ} \mathrm{C}$ in the dark to minimize degradation.

\section{(ii) Serum extraction}

A $200 \mu \mathrm{l}$ aliquot of serum was transferred into a centrifuge tube by a pipette. 20 $\mu \mathrm{l}$ of mono-hexyl lutein was added to this portion of serum as an internal standard. The concentration of the internal standard was $56.81 \mathrm{ng}$ per $20 \mu \mathrm{l}$. The serum proteins were precipitated by the addition of $2 \mathrm{ml}$ of ethanol/deionized water $(50: 50, \mathrm{~V}: \mathrm{V})$. The carotenoids were extracted with three $2 \mathrm{ml}$ portions of hexane, and followed by a $1 \mathrm{~min}$ vortex and 5 min centrifugation. The hexane layer was removed by pipette into a highdensity polypropylene vial and evaporated to dryness under a stream of nitrogen. All the procedures were preformed carefully in a dim light condition. The extracted carotenoid sample was dissolved in $50 \mu$ of ethanol for HPLC analysis, or the dried extracted 
carotenoid sample was stored in the dark $-20^{\circ} \mathrm{C}$ prior to HPLC analysis. To minimize experimental errors, two replicate extracted carotenoid samples were prepared from each serum sample and two analyses were made for each by injecting two aliquots from each extraction. The average concentrations of the carotenoids were calculated from the four injections.

\section{(iii) Preparation of MHL internal standard}

The internal standard was chosen because of its chemical similarity to that of the analyte. It was previously shown that the internal standard and the analytes would have same percent recovery in the process of extraction and would behave similarly on the HPLC column. Both assumptions have been proved in a previous study in our group. Mono-hexyl lutein (MHL) was chosen as an internal standard for quantitative analysis of extracted carotenoids from serum in this experiment. It was synthesized as follows:

An acidified hexanol solution was prepared by adding $10 \mu \mathrm{l}$ concentrated $\mathrm{HCl}$ to $10 \mathrm{ml} \mathrm{hexanol.} \mathrm{About} 1 \mathrm{mg}$ of dry lutein (Kemin Foods, Inc.) was dissolved in this acidified alcohol solution. The mixture was stored at $4{ }^{\circ} \mathrm{C}$ for at least $24 \mathrm{hrs}$ to allow the reaction to reach completion. The structure of MHL is shown in Figure 28. Fifty ml of saturated $\mathrm{NaHCO}_{3}$ solution was transferred to the mixture to quench the reaction. The carotenoids were extracted with equal portions of $\mathrm{CH}_{2} \mathrm{Cl}_{2}$. The $\mathrm{CH}_{2} \mathrm{Cl}_{2}$ was evaporated by a rotary evaporator. The dried MHL was dissolved in ethanol and purified by a reversed-phase HPLC system (2)(LDC/Milton Roy solvent delivery system) which coupled with an SM4000 UV-Visible detector set at $451 \mathrm{~nm}$. A manual injector with a $250 \mu$ injection loop was installed in this HPLC system. The column used was a $250 \times 4.6$ mm Ultracarb C18 $3 \mu \mathrm{m}$ ODS (Phenomenex ${ }^{\mathrm{TM}}$ ). The flow rate was $1.0 \mathrm{ml} / \mathrm{min}$. The 
mobile phase used was $85 \%$ acetonitrile, $15 \%$ methanol, with $0.1 \%(\mathrm{~V}: \mathrm{V})$ of triethylamine added. The MHL peak was collected at a retention time of $40 \mathrm{~min}$ and dried under a stream of nitrogen gas. The dried MHL was re-dissolved in ethanol. The concentration of MHL was determined by the absorption at a wavelength of $451 \mathrm{~nm}$. The absorption coefficient of MHL is considered the same as lutein which is $250 \mathrm{Lg}^{-1} \mathrm{~cm}^{-1}$.

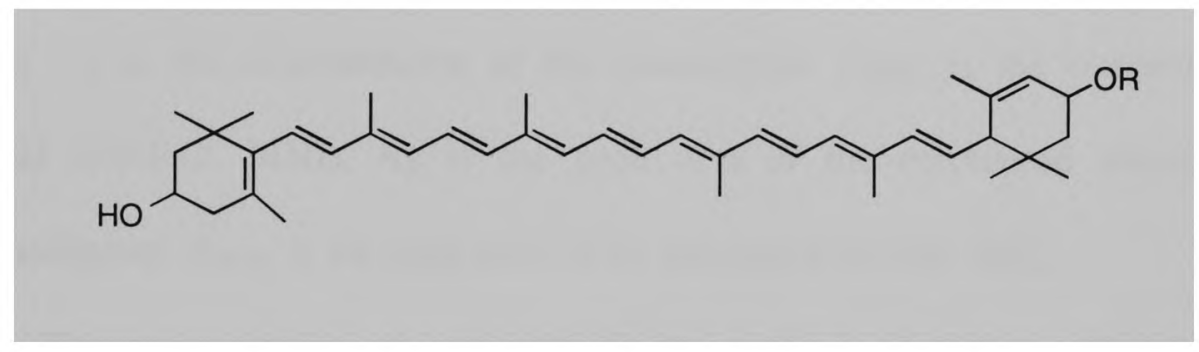

Figure 28. The structure of MHL. R=hexyl

\section{(iv) Reversed-phase HPLC conditions}

Analysis of all extracted carotenoids from serum samples were conducted on a Waters 2690 separation module HPLC with a Waters 486 UV tunable detector set at 451 nm. The data was stored and processed on the Millenium integration software. The column used was a $250 \times 4.6 \mathrm{~mm} \mathrm{C18}$ column packed with $3 \mu \mathrm{m}$ Ultracarb ODS (Phenomenex, Torrance, CA, U.S.A) and was protected with a C18 Phenomenex ${ }^{\mathrm{TM}}$ guard cartridge. Separation was achieved with a mobile phase of $85 \%$ acetonitrile and $15 \%$ methaol, with $0.1 \%(\mathrm{~V} / \mathrm{V})$ of triethylamine added to inhibit degradation of carotenoids during elution. The flow rate was $1.0 \mathrm{ml} / \mathrm{min}$.

\section{Results and Discussion}

The concentration of the carotenoids in serum are reported in $\mu \mathrm{g} / \mathrm{ml}$. The calculation is based on Beer's law 


$$
\mathrm{A}=\varepsilon \mathrm{bC}
$$

Where $\mathrm{A}$ is absorption, $\varepsilon$ is molar extinction coefficient, $\mathrm{b}$ is the pathlength of the cell, and $\mathrm{C}$ is the concentration of the analyte. The $\varepsilon$ value is the same for all analytes as well as the internal standard MHL. Therefore,

$$
\mathrm{C}_{\mathrm{A}}=\left(\mathrm{A}_{\mathrm{A}} \times \mathrm{C}_{\mathrm{MHL}}\right) / \mathrm{A}_{\mathrm{MHL}}
$$

Where $\mathrm{C}_{\mathrm{A}}$ is the concentration of the carotenoids. $\mathrm{C}_{\mathrm{MHL}}$ is the concentration of the internal standard, MHL. $\mathrm{A}_{\mathrm{A}}$ is the peak area of the carotenoid obtained from the chromatogram. $\mathrm{A}_{\mathrm{MHL}}$ is the peak area of the internal standard, MHL.

The concentration of zeaxanthin in the serum is plotted as a function of time as shown in Figure 29. It represents the average concentration of zeaxanthin of four replicate analyses vs the day of the supplementation. The first day of the supplementation is counted as zero.

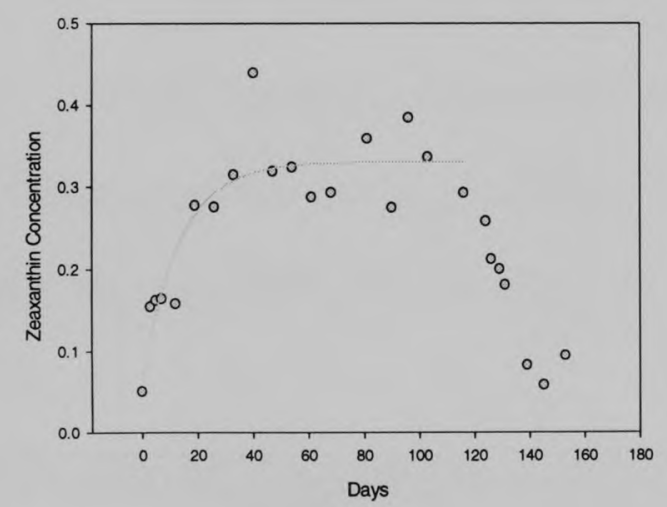

(a)

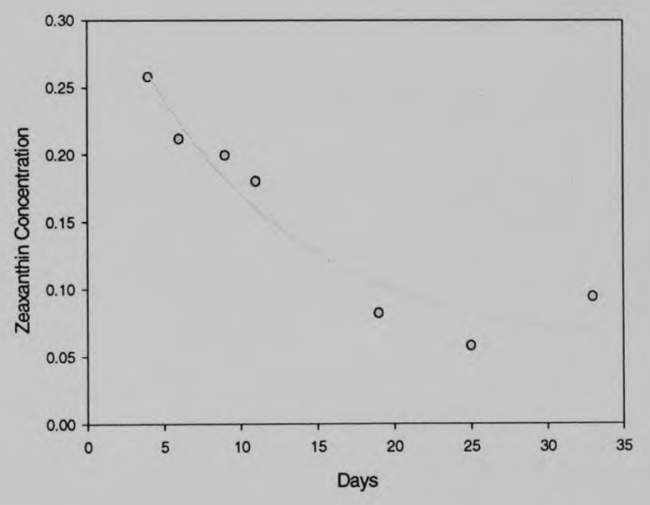

(b)

Figure 29. Plots of serum zeaxanthin concentration as a function of time. (a) represents the best fit during the supplementation, (b) represents the best fit after discontinue the supplementation. 
It can be seen that there is a significant increase of serum zeaxanthin during the first 40 days of supplementation. A plateau of about $0.30 \mu \mathrm{g} / \mathrm{ml}$ was maintained between the day 40-120 supplementation period. It was also found that the concentration dropped rapidly to the baseline level once the supplementation was discontinued. Although small changes were found in the concentration of zeaxanthin in serum due to the dietary sources, the effect of diet on this experiment is small because of the high dosage (30 $\mathrm{mg}$ per day) of zeaxanthin taken by the subject.

A baseline zeaxanthin concentration $\mathrm{C}_{0}$ was calculated from the average zeaxanthin concentration of pre-supplement analyses. The uptake and decay of zeaxanthin concentration in serum as a function of time were fit using equation (3) and (4), respectively. Those two equations have been also used to fit the experiment data in a previous long-term lutein supplementation study.

$$
\begin{aligned}
& C=C_{o}+a\left(1-e^{-k t}\right) \\
& C=C_{o}+b e^{-k^{\prime}\left(t-t_{f}\right)}
\end{aligned}
$$

Where $\mathrm{C}$ is the experimentally determined concentration. $t$ is the time. $t_{f}$ is equal to 120 days which is the first day when the subject discontinued the supplement. a, b, k, and k' are parameters varied to obtain the best fit. These fit parameters are found in table 2.

\begin{tabular}{|l|l|l|l|l|}
\hline PARAMETERS & ZEAXANTHIN & METABOLITE A & METABOLITE B & METABOLITE C \\
\hline $\mathrm{a}$ & 0.269 & 0.0038 & 0.0062 & 0.078 \\
\hline $\mathrm{b}$ & 0.029 & & 0.052 & 0.11 \\
\hline $\mathrm{k}$ & 0.071 & 0.067 & 0.054 & 0.041 \\
\hline $\mathrm{k}^{\prime}$ & 0.0566 & & 0.073 & 0.053 \\
\hline
\end{tabular}

Table 2. parameters for uptake and decay of zeaxanthin supplement and metabolite serum concentrations. 
During the supplementation, the metabolites of zeaxanthin were also determined. Their concentrations were calculated using the same method as that of zeaxanthin. Figure 30 (a) and (b) show the chromatograms of serum extract before and after 40 days supplementation.

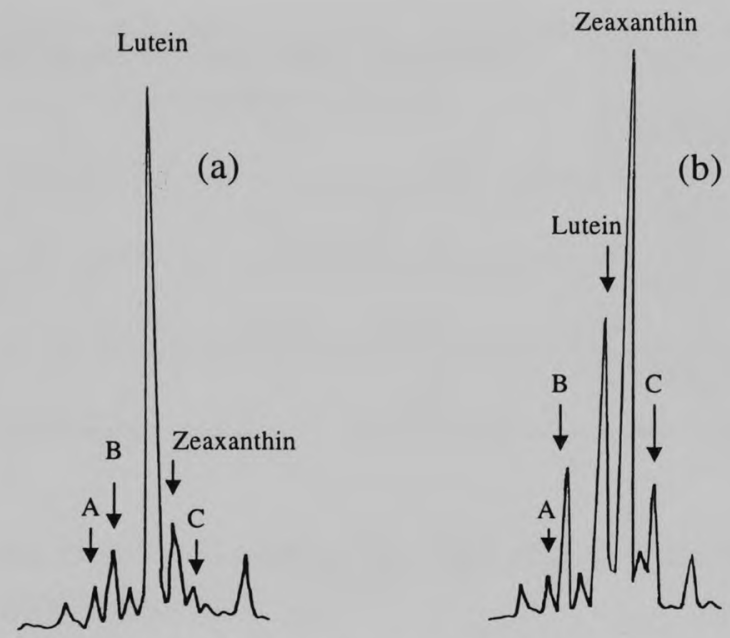

Figure 30. HPLC chromatogram of carotenoids from serum extract prior supplementation (a) HPLC chromatogram of carotenoids from serum extract after 40 days supplementation with $30 \mathrm{mg} /$ day zeaxanthin

Peaks A, B and C represent the xanthophyll metabolites that are observed in serum. Compound $\mathrm{C}$ has been characterized as a cis-isomer of zeaxanthin. It was found that both peak B and C increased significantly after supplementation with zeaxanthin. Figures 31,32 and 33 represent the average concentrations of these metabolites vs the days of the supplementation. The results show that metabolites B and C increase rapidly from the baseline levels of 0.03 , and $0.008 \mu \mathrm{g} / \mathrm{ml}$ to a plateau that is about three and ten times higher, respectively, than the baseline level prior to the first 40 days 
supplementation. The concentrations of both $\mathrm{B}$ and $\mathrm{C}$ dropped to the baseline level once the supplementation was discontinued. The average concentration of metabolite A did not change during zeaxanthin supplementation. It maintains an average concentration of $0.016 \mu \mathrm{g} / \mathrm{ml}$. This result supports those obtained previously that suggest A to be a lutein metabolite. The changes in the average concentrations of zeaxanthin metabolites A, B and $\mathrm{C}$ are listed in table 3.

\begin{tabular}{|l|c|c|}
\hline Average concentration (Standard Deviation) & Baseline Level & Plateau Level \\
\hline Zeaxanthin & $0.055( \pm 0.007)$ & $0.324( \pm 0.053)$ \\
\hline Metabolite A & $0.016( \pm 0.003)$ & $0.016( \pm 0.002)$ \\
\hline Metabolite B & $0.03( \pm 0.003)$ & $0.08( \pm 0.007)$ \\
\hline Metabolite C & $0.008( \pm 0.0003)$ & $0.077( \pm 0.006)$ \\
\hline
\end{tabular}

Table 3. Serum average concentration and standard deviation of zeaxanthin and the metabolites A, B and C

The calculated half-lives for the uptake and decay of zeaxanthin and metabolites, and the half-lives of lutein obtained from a previous experiment are listed in table 4.

\begin{tabular}{||l|l|l|l|l||}
\hline \hline HALF-LIVES & LUTEIN & ZEAXANTHIN & METABOLITE B & METABOLITE C \\
\hline $\mathrm{t}_{1 / 2}$ uptake & 9.6 & 9.76 & 12.83 & 16.90 \\
\hline $\mathrm{t}_{1 / 2}$ decay & 8.13 & 12.24 & 9.49 & 13.08 \\
\hline
\end{tabular}

Table 4. The calculated half-lives for the uptake and decay of the concentrations of lutein, zeaxanthin and metabolites $\mathrm{B}$ and $\mathrm{C}$. 


\section{Conclusion}

The concentration of zeaxanthin as well as the metabolites $\mathrm{B}$ and $\mathrm{C}$ in human serum is increased by zeaxanthin supplementation during the first 40 days of supplementation. Plateaus maintained concentrations of $0.32,0.079$, and $0.077 \mu \mathrm{g} / \mathrm{ml}$ between days 40 - 120 of supplementation for zeaxanthin and metabolites B and C, respectively. The concentrations dropped to the baseline level once the supplement was discontinued. No correlation was found for metabolite A during zeaxanthin supplementation.

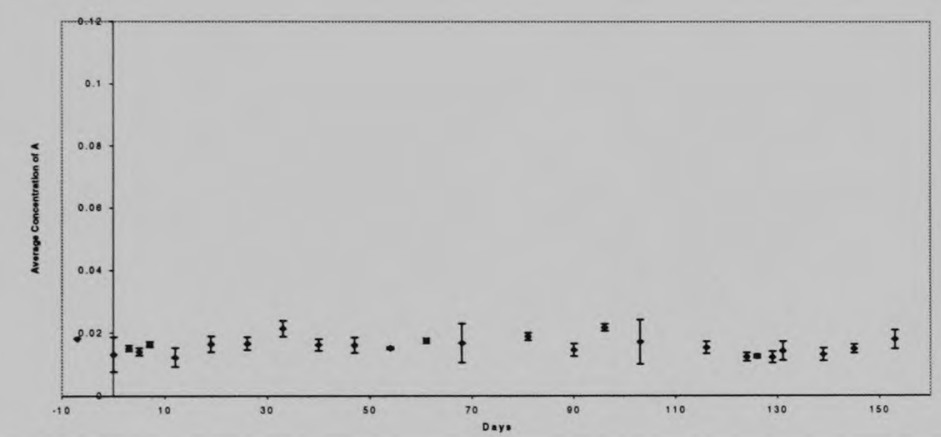

Figure 31 . The plot of serum concentration of metabolite $\mathrm{A}$ as a function of time.

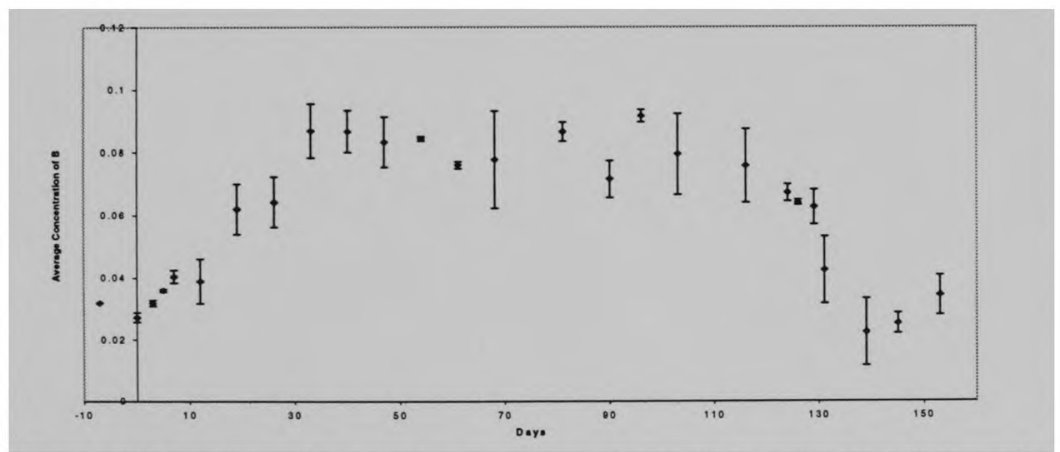

Figure 32. The plot of serum concentration of metabolite B as a function of time. 




Figure 33. The plot of serum concentration of metabolite $\mathrm{C}$ as a function of time. 


\section{PART 3 A SIX MONTH STUDY OF LUTEIN UPTAKE IN 22 HUMAN SUBJECTS}

\section{Overview}

Lutein, as a micronutrient, has been found in many fruits and vegetables that we consume. It is also one of the most abundant carotenoids found in the human body. It acts as an antioxidant. The structure of lutein is shown in Figure 1.

As an antioxidant nutrient, lutein is normally accumulated in the macula region of the human retina. The macula is the anatomical region of the retina that is responsible for central vision. It is composed of several functionally and structurally distinct layers overlaying and supporting the cone and rod photoreceptors. Lutein and zeaxanthin are the major constituents of human macular pigment. (Bone. et al., 1985). Macular lutein is believed to function as a blue light filter by absorption of blue light. The macular carotenoids can reduce the blue light intensity reaching photoreceptors on average by about 40 percent. (Landrum, et al., 1996) Blue light can cause the formation of singlet oxygen, triplet excited state species, and free radicals. Lutein may act as an active quencher of singlet oxygen, and excited state triplets. It can react with and deactivate radical species preventing chain propagation. These actions may all be functions of carotenoids of the macular pigment.

Age - related macular degenaration (AMD) is one of the eye diseases which disrupts the normal functioning of the macula. It occurs in about $20 \%$ of people over the age of 65. (Landrum, et al., 1995; Landrum, et al., 1997) It is an irreversible condition of 
a visual impaiment. There are many factors with the positive correlation with AMD. The low levels of dietary xanthophylis and serum xanthophylis are two factors among them. Landrum, et al., (1995) have reported that lower than normal amounts of macular pigment are found in person with AMD. The macular pigment may reduce the risk of the AMD. (Hyman, 1992, Eye Disease Case-Control Study Group, 1993, Seddon, et al., 1994) In Landrum's early study, it is reported that the amount of the macular pigment may be enhanced by dietary supplementation with $30 \mathrm{mg}$ of lutein per day. (Landrum, et $a l ., 1997)$ It is known that the average optical density of the macular pigment in the retina is proportional to the average pigment concentration. (Handelman, et al., 1991) The optical density can be measured by heterochromatic flicker photometry. It was established that the macular pigment optical density increased in the eyes when the concentration of lutein was elevated in the serum during a long-term supplementation study with a lutein ester form supplement. (Landrum, et al 1997) It was found that the concentration of lutein in serum decreased and returned to pre-supplementation levels after the subjects discontinued the supplement. However, the optical density remained higher than the pre-supplementation level after conclusion of supplementation and did not return to baseline. Therefore, the hypothesis that lower doses of lutein might also cause similar increases in macular pigment optical density in eyes was suggested. This study, based on the earlier methods, used a large group of subjects (22 human subjects) in order to determine if low dosages produced a significant result. 


\section{Experimental}

(1) Reagents and materials

HPLC grade Methanol, Acetonitrile, Hexane and Ethanol were purchased from Fisher. Nitrogen gas was purchased from Air Products and Chemicals, Inc.

A $250 \times 4.6 \mathrm{~mm}$ column Ultracarb C18 $3 \mu \mathrm{m}$ ODS and a guard column were from the Phenomenex, Torrance, CA, U.S.A.

(2) Apparatus

The reversed phase HPLC was a Waters 2690 separation module equipped with a column heater and a sample cooling system. A Waters 486 tunable absorbance UV/Vis detector was used for detection.

A heterochromatic flicker photometer was constructed as previously described. (Bone, et al., 1971, Bone, et al., 1992)

(3) Procedures

In this study, 22 healthy, non-smoker human volunteers were selected. None of the subjects took a commercial dietary supplement that included carotenoids during the period of time of this study. Eye exams were performed by an ophthalmologist before supplementation for each subject. For several subjects a follow-up eye exam was also performed. These 22 volunteers supplemented their diets with lutein $(1.2 \mathrm{mg} \times 2$ per day), in the form of Nutrilite's softgel Bilberry with Lutein Botanical Blend for a period of six months. Blood samples were drawn weekly from the subjects and analyzed by reversed-phase HPLC. The initial baseline of serum lutein level and postsupplementation serum lutein level were monitored for the subjects. The same experimental method as previously described was conducted. The macular pigment 
optical density for the subjects was measured twice each week by heterochromatic flicker photometry to determine both pre- and post- optical density levels in their eyes.

\section{(i) Serum sampling and handling}

Blood was sampled into $5 \mathrm{ml}$ Vacutainer serum separator tubes by venipuncture. Serum was separated from blood and stored in the freezer by the same procedures as described in part 2. Serum samples were labeled to identify the subjects and the day the blood was sampled.

\section{(ii) Serum extraction}

Serum extraction was preformed by following the same procedures as in part 2 . A $200 \mu \mathrm{l}$ aliquot of serum was taken for the extraction from each serum sample. $20 \mu \mathrm{l}$ of monhexyl lutein (MHL) was added to this portion of serum as an internal standard. The amount of the internal standard was about $50 \mathrm{ng}$. The extracted sample was dissolved in a $50 \mu$ of ethanol for HPLC analysis, or the dried extracted sample was stored in the dark at $-20^{\circ} \mathrm{C}$ until HPLC analysis. A replicate injection was preformed for the each extraction in order to minimize the experimental errors.

\section{(iii) HPLC conditions}

All serum samples were analyzed on a Waters 2690 module HPLC with a Waters $486 \mathrm{UV}$ tunable detector set at $451 \mathrm{~nm}$. The data were stored and processed on Millenium intergration software. The column used was a $250 \times 4.6 \mathrm{~mm} \mathrm{C18}$ column with a C18 guard column packed with $3 \mu \mathrm{m}$ Ultracarb ODS. The column was housed in a column heater set at $25^{\circ} \mathrm{C}$. A sample cooling system was installed in this HPLC and it was set to $4^{\circ} \mathrm{C}$ to minimize potential sample degradation. Separation was achieved with a mobile 
phase of $80 \%$ acetonitrile and $20 \%$ of methaol, with $0.1 \%(\mathrm{~V} / \mathrm{V})$ of triethylamine added to inhibit degradation of carotenoids during elution. The flow rate was $1.0 \mathrm{ml} / \mathrm{min}$.

\section{Results and discussion}

The pre- and post- supplementation serum lutein levels and macular pigment optical densities of the subjects are shown in Table 5. The baseline serum lutein concentrations and macular pigment optical densities were measured as the mean value of all measurements during the period of time of pre- supplementation. The first day of supplementation was counted as zero. The post- supplementation serum lutein concentrations and macular optical densities were the mean value of all measurements taken between day 7 and day 150 of supplementation. For the subjects who dropped-out from the study prior to day 150 , the mean concentration and macular pigment optical densities were calculated from all data after day 7. It was observed that there was a significant increase in serum lutein concentration for all subjects. The percentage increase ranged from $20 \%$ to $300 \%$ with a mean increase of $128 \%$ (median $=116 \%)$. The increase in macular pigment optical density was significant for 11 of the 19 subjects. The mean increase for all 19 subjects was also significant as $4.5 \%$.

Baseline dietary intake for lutein and zeaxanthin of $1.2 \pm 1.1 \mathrm{mg} / \mathrm{day}$ was determined from a food frequency questionnaire. Therefore, the $2.4 \mathrm{mg}$ of dosage represents about twice the mean dietary intake. Figure 34 shows the plot of presupplement serum lutein level vs dietary intake. A significant correlation was found showing that the higher the dietary intake the higher the supplementation level of preserum lutein. We also note that individuals with high pre-supplementation serum lutein levels also had high post-supplementation levels (Figure35). Figure 36 (a) and (b) 
represent two typical examples from the two subjects with different pre- supplementation serum lutein levels. Subject B has higher pre- and post- supplementation serum lutein levels than that of subject A.

\begin{tabular}{|c|c|c|c|c|c|c|c|c|}
\hline \multirow[b]{2}{*}{$\begin{array}{l}\text { Subject } \\
\text { number }\end{array}$} & \multicolumn{4}{|c|}{ Serum Lutein $(\mu \mathrm{g} / \mathrm{ml})$} & \multicolumn{4}{|c|}{ Macular Pigment Optical Density } \\
\hline & $\begin{array}{l}\text { Pre- } \\
\text { supplement }\end{array}$ & $\begin{array}{l}\text { Post- } \\
\text { supplement }\end{array}$ & \%increase & $\begin{array}{l}\text { Absolute } \\
\text { increase }\end{array}$ & $\begin{array}{l}\text { Pre- } \\
\text { supplement }\end{array}$ & $\begin{array}{l}\text { Post- } \\
\text { supplement }\end{array}$ & \%increase & $\begin{array}{l}\text { Absolute } \\
\text { increase }\end{array}$ \\
\hline 1 & 0.092 & 0.137 & 49 & 0.045 & 0.415 & 0.426 & 2.8 & 0.011 \\
\hline 2 & 0.187 & 0.384 & 106 & 0.197 & 0.448 & 0.495 & 10.5 & 0.047 \\
\hline 3 & 0.104 & 0.275 & 165 & 0.171 & 0.678 & 0.695 & 2.5 & 0.017 \\
\hline 4 & 0.091 & 0.284 & 211 & 0.192 & 0.28 & 0.4 & 40 & 0.12 \\
\hline 5 & 0.076 & 0.148 & 95 & 0.072 & 0.397 & 0.413 & 4.2 & 0.016 \\
\hline 6 & 0.163 & 0.382 & 134 & 0.218 & 0.315 & 0.338 & 7.5 & 0.023 \\
\hline 7 & 0.257 & 0.339 & 32 & 0.082 & 0.81 & 0.83 & 2 & 0.02 \\
\hline 8 & 0.058 & 0.132 & 127 & 0.074 & 0.243 & 0.252 & 3.7 & 0.009 \\
\hline 9 & 0.188 & 0.336 & 79 & 0.148 & 0.6 & 0.61 & 1 & 0.01 \\
\hline 10 & 0.062 & 0.179 & 189 & 0.117 & 0.205 & 0.219 & 6.8 & 0.014 \\
\hline 11 & 0.059 & 0.231 & 291 & 0.172 & 0.311 & 0.311 & 0 & $\mathbf{0}$ \\
\hline 12 & 0.141 & 0.459 & 225 & 0.317 & 0.612 & 0.65 & 6.1 & 0.038 \\
\hline 13 & 0.148 & 0.307 & 107 & 0.159 & 0.384 & 0.487 & 27 & 0.103 \\
\hline 14 & 0.163 & 0.292 & 79 & 0.129 & 0.45 & 0.514 & 14 & 0.064 \\
\hline 15 & 0.250 & 0.311 & 25 & 0.061 & 0.387 & & & \\
\hline 16 & 0.089 & 0.210 & 136 & 0.121 & 0.613 & 0.706 & 15 & 0.093 \\
\hline 17 & 0.203 & 0.275 & 35 & 0.071 & 0.465 & 0.489 & 5 & 0.024 \\
\hline 18 & 0.208 & 0.469 & 126 & 0.261 & 0.307 & 0.388 & 26 & 0.081 \\
\hline 19 & 0.080 & 0.340 & 325 & 0.260 & 0.186 & 0.318 & 71 & 0.132 \\
\hline 20 & 0.240 & 0.280 & 17 & 0.040 & 0.711 & 0.719 & 1 & 0.008 \\
\hline 21 & 0.060 & 0.202 & 235 & 0.142 & 0.478 & \begin{tabular}{|l|}
0.528 \\
\end{tabular} & 10.3 & 0.05 \\
\hline Average & & & 128 & & & & 4.5 & \\
\hline
\end{tabular}

Table 5. Pre- and post- supplementation serum lutein levels and macular pigment optical densities of the subjects

We can see that one of the metabolites, labeled as peak A, increased significantly during the supplementation. (Figure7) Table 6 shows average \% increases in several serum carotenoid levels. Some variation of serum carotenoid levels occured during the period of time of supplementation since the subjects were on an uncontrolled diet. Peak A 
is the only significant change other than lutein in the chromatogram. This agrees with the results of the high dosage lutein supplementation study and gives further evidence that $\mathrm{A}$ is the metabolite of lutein. However, B and C only had little increases. We noted that different forms of lutein supplement have been taken in this and previous studies. Instead of a form of supplement containing natural free lutein, a commercial available supplement form was giving. It is understandable that the different form of the supplement should affect the presence of the metabolites.

\begin{tabular}{|l|l|}
\hline & \% Average increase \\
\hline Lutein & 128 \\
\hline Macular Pigment Optical Density & 12.8 \\
\hline Peak A & 101.2 \\
\hline Peak B & 24.3 \\
\hline Peak C & 36.4 \\
\hline Zeaxanthin & 12.9 \\
\hline$\beta$-Cryptoxanthin & 3.6 \\
\hline
\end{tabular}

Table 6. Percent average increases from all human subjects in several carotenoids, metabolites and optical density after supplementation

\section{Conclusion}

This study has shown that supplementation of the normal diet with as little as $2.4 \mathrm{mg} / \mathrm{day}$ of lutein results in increased serum levels of lutein by as much as $300 \%$ in some individuals and results in a increase in macular pigment optical density for 19 of the study group. We also noted that the serum levels of several metabolites also have increases. A correlation between pre-supplementation and post-supplementation was also 
found. The higher the pre-supplementation level the higher the post-supplementation level of serum lutein.

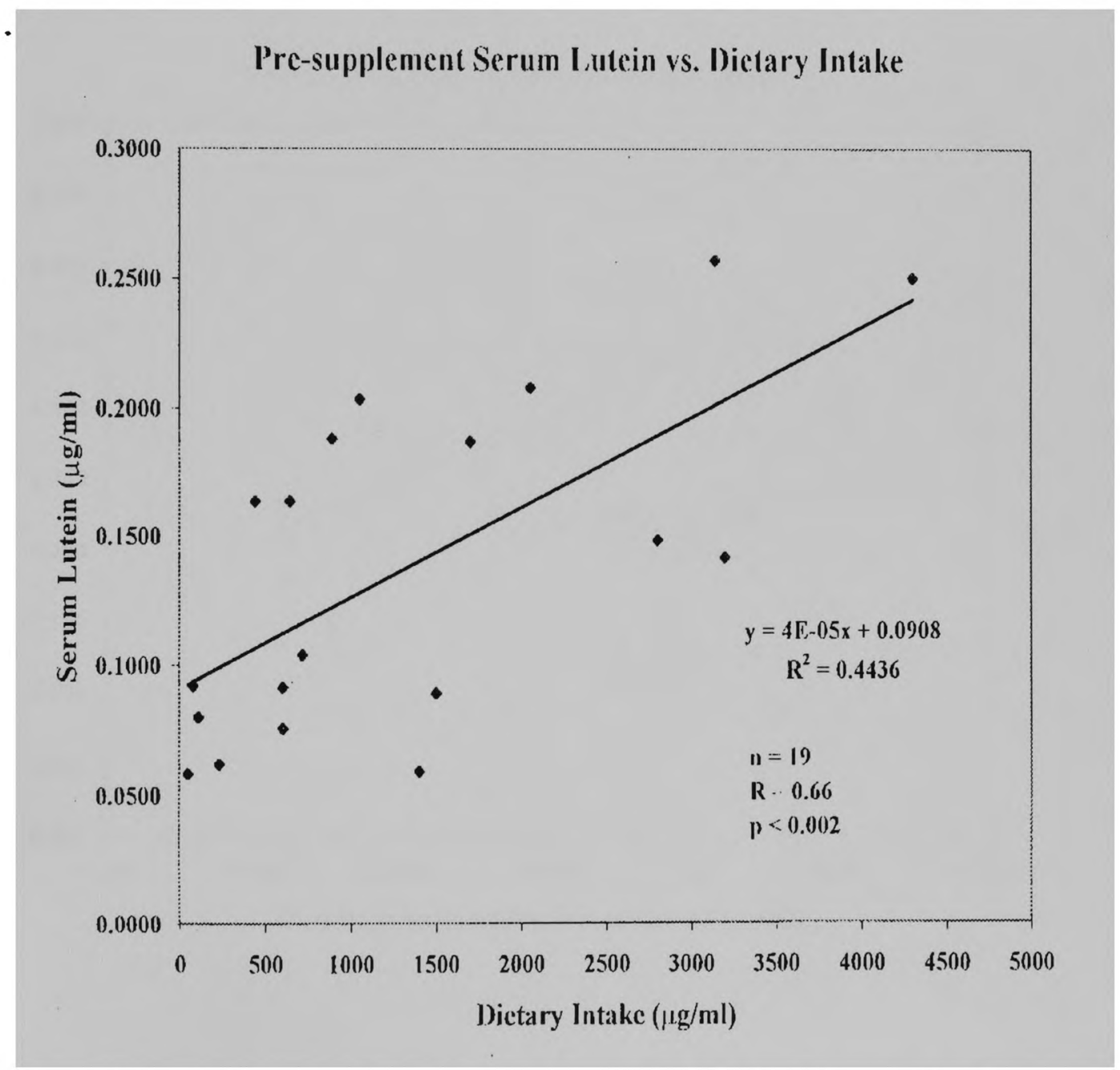

Figure 34. Plot of dietary intake vs the concentration of serum lutin 


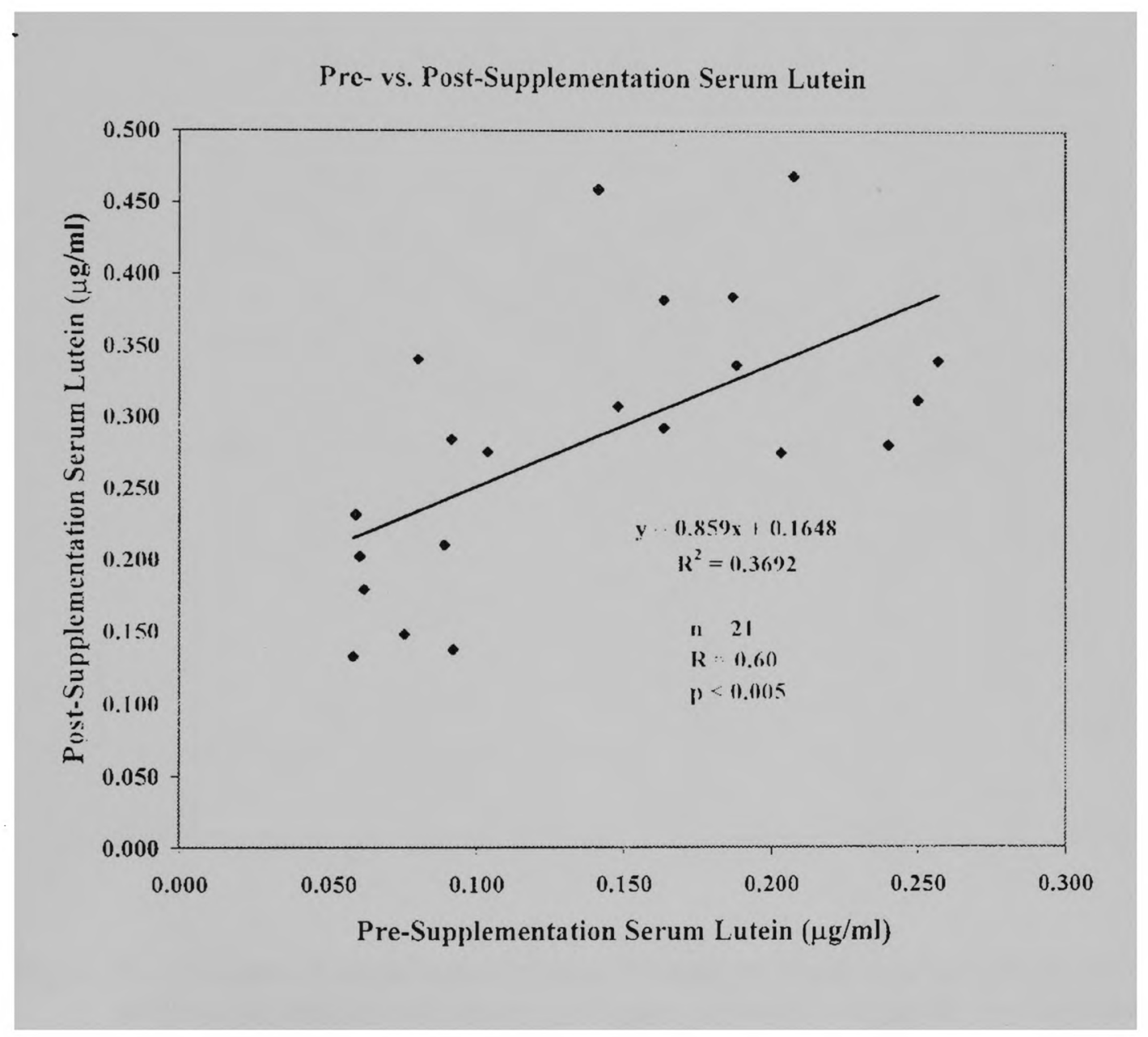

Figure 35. Plot of pre-supplementation serum lutein concentration vs postsupplementation serum lutein concentration 
(a)

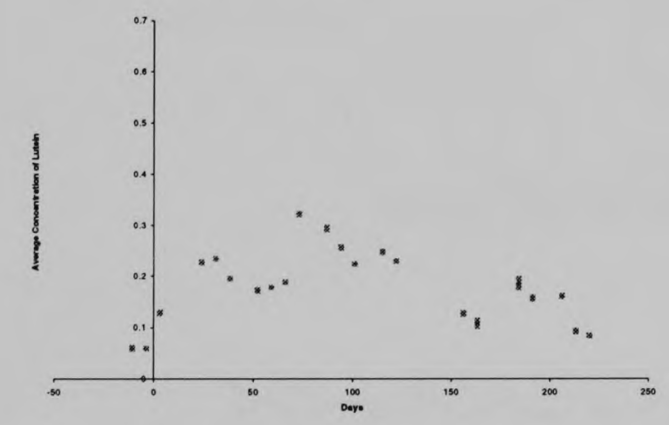

(b)

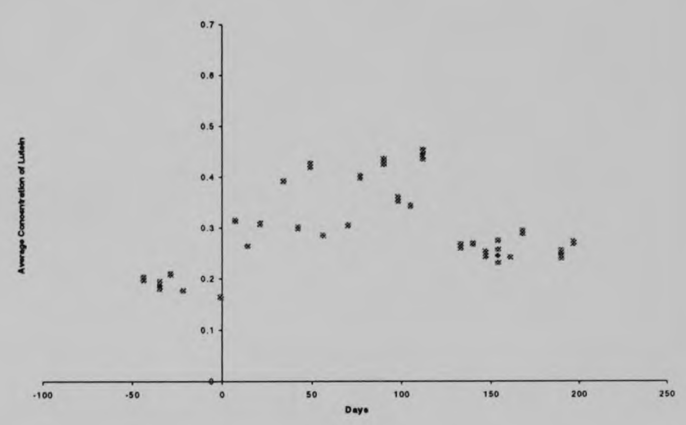

Figure 36. The plots of serum concentration of lutein as function of time from two human subjects (a) and (b) with lower and higher pre-supplementation serum lutein levels. 
IV PART 4

\section{SAMPLES}

\section{Overview}

Zeaxanthin is one of the carotenoids found in the human body. It has two stereo centers in the positions of $\mathrm{C} 3$ and $C 3^{\prime}$ ' which give rise to three stereoisomers, (3R, $\left.3^{\prime} \mathrm{R}\right)$ $\beta, \beta$-carotene-3,3'-diol, (3R,3'S)- $\beta, \beta$-carotene-3,3'-diol and (3S,3'S)- $\beta, \beta$-carotene-3,3'diol. The structures of these three stereoisomers are illustrated in Figure 37.<smiles></smiles>

\section{Zeaxanthin}

$\left(3 R, 3^{\prime} R\right)-\beta, \beta$-carotene-3,3'-diol<smiles>CC1=C(/C=C/C(C)=C/C=C/C(C)=C/C=C/C=C(C)/C=C/C=C(C)/C=C/C2=C(C)[C@H](O)CC2(C)C)C(C)(C)C[C@@H](O)C1</smiles>

meso-Zeaxanthin

(3R,3'S)- $\beta, \beta$-carotene-3,3'-diol<smiles>CC1=C(/C=C/C(C)=C/C=C/C(C)=C/C=C/C=C/C(C)=C/C=C/C=C(C)/C=C/C2=C(C)[C@H](O)CC2(C)C)C(C)(C)C[C@H](O)C1</smiles>

(3S, 3'S)-Zeaxanthin

$\left(3 S, 3^{\prime} S\right)-\beta, \beta$-carotene-3,3'-diol

Figure 37. Stereochemical structures of zeaxanthin 
As mentioned earlier, lutein and zeaxanthin are major components of macular pigment in the human retina. (Bone, et al., 1988) The macular pigments play a protective role in the eye. The concentration of zeaxanthin is about twice that of lutein in the inner macula. (Bone, et al., 1988) The macula contains the highest density of cone photoreceptors and is specialized for acute central vision. The macula is visually pigmented with the yellow carotenoids. The yellow spot is about 2 to $3 \mathrm{~mm}$ in diameter in human eyes. The macula is divided into the inner macula and the outer macula. The inner macula is about $1.5 \mathrm{~mm}$ in diameter and the outer macula is the surrounding annulus. Figure 38 shows the human eye in cross-section. As eccentricity from the fovea increases, the lutein/zeaxanthin ratio of concentrations continuously changes with lutein becoming the dominant component. It was reported that all three zeaxanthin stereoisomers (RR, RS, SS) are present in the human eye. (Landrum, et al., 1999; Bone, et al., 1997) The concentration of RS zeaxanthin decreases with eccentricity. This decrease in RS zeaxanthin closely corresponds to the increase in lutein. (Landrum, et al., 1999; Bone, et al., 1997) Among the three zeaxanthin stereoisomers, the RR configuration is the only one that is found in significant quantities in the diet and is relatively abundant in a number of fruits and corn. Since animals cannot synthesize carotenoids, RR-zeaxanthin would be expected to be the sole stereoismer to be found in human body. The presence of RS and SS zeaxanthin in the retina suggests that the conversion of lutein to zeaxanthin may occur by migration of a double bond in the lutein molecule. Landrum et al have reported that only RR-zeaxanthin has been detected in human blood. (Landrum, et al., 1999) Therefore, the process of conversion would seem 
to occur in the eye. The purpose of this study was to confirm the absence of RS and SS zeaxanthin in human serum. The composition of zeaxanthin stereoisomers in serum

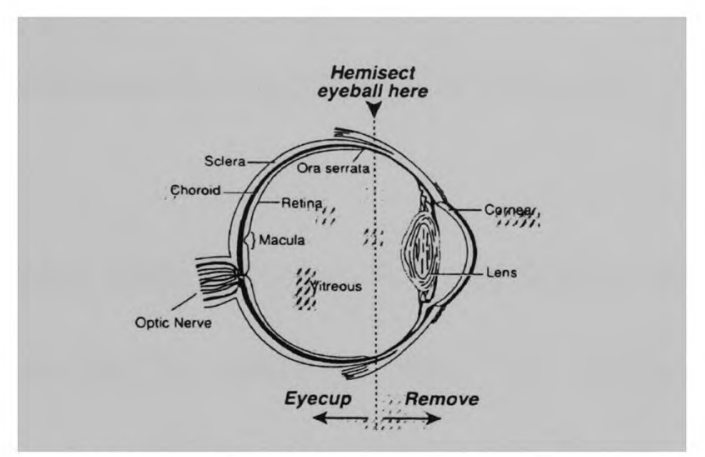

Figure 38. Cross-sectional diagram of the human eye. (Handelman, 1992)

samples from 18 subjects was studied by normal phase HPLC.

\section{Experimental}

(1) Reagents and materials

HPLC grade Methanol, Acetonitrile, Hexane, Ethanol, and Isopropyl acetate were purchased from Fisher. Pyridine anhydrous $99 \%$ (water $<0.005 \%$ ), benzene $(99.8 \%$ anhydrous), and (S)-(+)-1-(1-Naphthyl) ethyl isocyanate were purchased from Aldrich Chemical Company. (Milwaukee WI 53233 USA) Synthesized, pure RR, RS, and SS zeaxanthin were obtained as a gift from the company Hoffman La Roche.

A $250 \times 2.0 \mathrm{~mm}$ normal phase column packed with $5 \mu \mathrm{m}$ Prodigy silica and a $250 \times 10.0 \mathrm{~mm}$ reversed phase column packed with $3 \mu \mathrm{m}$ Ultracarb ODS were purchased from Phenomenex, Torrance CA. U.S.A. 
(2) Apparatus

Reversed phase HPLC system (1) was an LDC Analytica Consta Metric 3200 solvent delivery system equipped with a $50 \mu \mathrm{l}$ injection loop and was coupled with an LDC/Milton Roy UV detector. Normal phase HPLC, system (2), was an LDC Milton Roy system which included an SM4000 UV/Vis detector.

(3) Procedures

Serum samples were obtained from 20 human subjects. $2 \mathrm{ml}$ of serum from each subject was extracted. In order to isolate the zeaxanthin from each sample, reversedphase HPLC system (1) was employed. This included a $250 \times 10.0 \mathrm{~mm} \mathrm{C18}$ column. Zeaxanthin was collected during elution and dried under a stream of nitrogen gas. The dicarbamate derivatization reaction was carried out individually on the collected zeaxanthin for each subject's sample. The dicarbamate derivatives of the collected zeaxanthin were further analyzed by normal phase HPLC, system (2), to determine the stereoisomer composition. The separated stereoisomer peaks were collected and dried for further identification analysis. For comparison purposes, a racemic standard of the three synthesized zeaxanthin stereoisomer mixtures was converted to the dicarbamate derivatives. The concentration ratio of these three stereoisomers, RR:RS:SS, was equal to 1:2:1. The identification of zeaxanthin stereoisomers in 20 human subjects was determined from the peak enhancement by the co-injection of the collected, derivatized stereoisomer components with the racemic mixture of zeaxanthin dicarbamates.

\section{(i) Preparation of standard}

Three synthetic zeaxanthin steroisomers, (3R,3'R)- $\beta, \beta$-carotene-3,3'-diol, (3R,3'S)- $\beta, \beta$-carotene-3,3'-diol and (3S,3'S)- $\beta, \beta$-carotene-3,3'-diol, were obtained as a 
gift from the company Hoffman-La Roche. They were separately dissolved in EtOH in three pear-shaped flasks. Since the absorption coefficients of these three stereoisomers are identical, the concentration ratio (RR:RS:SS) was determined from UV spectrometry. The three stereoisomers were mixed together to produce a concentration ratio of RR:RS:SS equal to $1: 2: 1$. This racemic-mixture of steroisomers was dried under a stream of nitrogen gas. The dicarbamate derivatization reaction was carried out under nitrogen gas in a glove box following the derivatization procedures described below.

\section{(ii) Carotenoids extraction and zeaxanthin collection}

$2 \mathrm{ml}$ of serum from each subject was extracted individually by following the extraction procedures mentioned previously. The extracted carotenoids were dissolved in $200 \mu \mathrm{l} \mathrm{EtOH}$ for reversed-phase HPLC separation. All carotenoid separations were conducted on reversed-phase HPLC system (1) with a UV detector set at $451 \mathrm{~nm}$. A C18 $250 \times 10 \mathrm{~mm}$ reversed phase column was used for the separation. A mobile phase of $85 \%$ acetonitrile, $15 \%$ methanol and with $0.1 \%$ triethlyamine was used. The flow rate was 4.0 $\mathrm{ml} / \mathrm{min}$. The zeaxanthin peak was collected in a plastic vial, having been identified from its retention time which was known by running a lutein and zeaxanthin (1:1) standard in the same HPLC system. The collected zeaxanthin was dried under nitrogen gas for subsequent derivatization.

\section{(iii) Zeaxanthin dicarbamate derivatization reaction}

The plastic vial with the dried zeaxanthin from serum was transferred into a glove box containing a dry nitrigen atmosphere in order to carry out the derivatization reaction. The zeaxanthin was dissolved in $50 \mu \mathrm{l}$ of anhydrous pyridine/benzene (50:50 v/v). $2 \mu \mathrm{l}$ of 
(S)-(+)-1-(1-naphthyl) ethly isocyanate was added to the above solution and the mixture was capped and centrifuged for $30 \mathrm{sec}$. The plastic vial was covered with aluminum foil to exclude light and the mixture was allowed to react for at least $48 \mathrm{hr}$. Figure 40 shows the chemical reaction.

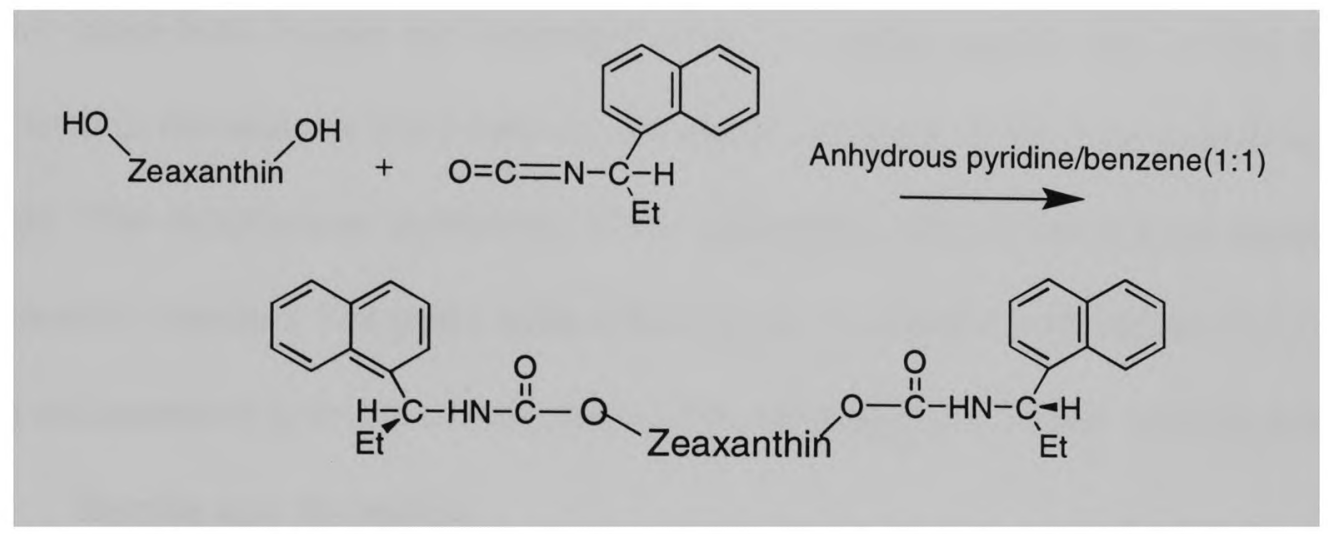

Figure 39. The chemical reaction of zeaxanthin dicabamate derivatization

The resulting dicarbamate derivatives were removed from the glove box. $5 \mathrm{ml}$ of wet (saturated) hexane was used to rinse the plastic vial and transfer the reaction mixture to a centrifuge tube. The solution becomes very cloudy. The derivatized zeaxanthin was extracted by addition of three $5 \mathrm{ml}$ portions of hexane, followed by $1 \mathrm{~min}$ vortex, $5 \mathrm{~min}$ centrifugation. The top clear portion of hexane was removed carefully to a pear-shaped flask by a syringe and dried under a stream of nitrogen gas. Since the amount of zeaxanthin was very small all of the procedures were carried out very carefully in order to prevent any loss.

\section{(iv) Normal phase HPLC separation of zeaxanthin stereoisomers}

The dicarbamate zeaxanthin derivatives were dissolved in a $20 \mu \mathrm{l}$ of the mobile phase and analyzed by a normal phase HPLC (LDC solvent delivery system) coupled 
with a UV detector. A $250 \times 2.0 \mathrm{~mm}$ normal phase column packed with $5 \mu \mathrm{m}$ silica (Phenomenex ${ }^{\mathrm{TM}}$ ) was used. The mobile phase was $88 \%$ hexane and $12 \%$ isopropyl acetate at a flow rate of $0.2 \mathrm{ml} / \mathrm{min}$. Detection was set at $451 \mathrm{~nm}$.

In order to obtain a good separation, the mobile phase solvent was prepared freshly since both hexane and isopropyl acetate evaporate rapidly. No internal standard was used in the analysis since only the relative proportions of the three stereoismers was sought. The dicarbamate derivatives of the zeaxanthin stereoisomers were compared to the racemic standard. The peaks were collected and co-injected with the racemic mixture. Peak enhancement provides a reasonably good confirmation of isomer identification.

\section{Results and discussion}

The normal phase HPLC chromatogram of racemic mixture standard is shown in Figure 40. Three zeaxanthin stereoisomers are well separated. The elution order of the three zeaxanthin stereoisomers is SS, RS and RR with retention time of $36.11 \mathrm{~min}, 38.85$ min and $42.48 \mathrm{~min}$, respectively. The results of the normal phase separation of the derivatized macular pigment zeaxanthin isomers from 18 human subjects are presented in Table 7 and show the percentage of the SS, RS and RR in serum zeaxanthin. It is clear that $\mathrm{RR}$ confirmation is the principal isomer. Figure 41 is a normal phase HPLC chromatogram from 20 human subjects showing the presence of a major RR peak. This result supports the hypothesis that the lutein to meso-zeaxanthin conversion process occurs in the eye. For further identification, the principal peak was collected and combined with the racemic mixture. An identical result was obtained for all 18 human subjects. Figure 42 is a typical example of the co-injection HPLC chromatogram of the collected stereoisomer peak with the zeaxanthin standard racemic mixture. The observed 
enhancement of the all E-R,R-zeaxanthin dicabamate peak provides us the most reliable conclusion that serum is composed chiefly of this isomer.

\begin{tabular}{|c|c|c|c|}
\hline SUBJECTS & $\%$ \%SS & $\% \mathrm{RR}$ & \%RS \\
\hline 1 & 0.00 & 1.27 & 98.9 \\
\hline 2 & 1.45 & 3.95 & 94.6 \\
\hline 3 & 0.00 & 3.20 & 96.7 \\
\hline 4 & 0.00 & 8.28 & 91.8 \\
\hline 5 & 1.05 & 3.42 & 95.5 \\
\hline 6 & 0.78 & 5.20 & 92.8 \\
\hline 7 & 0.00 & 6.96 & 93.1 \\
\hline 8 & 0.00 & 4.09 & 93.9 \\
\hline 9 & 0.00 & 11.7 & 88.4 \\
\hline 10 & 0.00 & 0.00 & 100 \\
\hline 11 & 0.00 & 0.00 & 100 \\
\hline 12 & 2.67 & 2.05 & 95.3 \\
\hline 13 & 0.00 & 1.24 & 98.8 \\
\hline 14 & 3.45 & 2.85 & 93.7 \\
\hline 15 & 0.00 & 3.20 & 96.8 \\
\hline 16 & 0.00 & 0.00 & 100 \\
\hline 17 & 0.00 & 0.00 & 100 \\
\hline 18 & 0.00 & 0.00 & 100 \\
\hline Average $\%$ & 0.52 & 3.19 & 96.3 \\
\hline
\end{tabular}

Table 7. Percentage of zeaxanthin stereoisomers in serum

A small peak can be observed in derivatized zeaxanthin samples of some human subjects. (Table 5) This is believed to be the RS-zeaxanthin component. The average percentage of the RS configuration of the total zeaxanthin is about $3 \%$. The SS- 
zeaxanthin isomer might be present in some samples based on the presence of a small peak at the correct elution time. The minute quantities of these potentially RS- and SSprevented confirmation by co-injection.

\section{Conclusion}

A significant difference in zeaxanthin stereoisomer composition between the serum and the retina was verified in this study. The determination of the composition of the zeaxanthin stereoisomers in 18 human subjects was carried out. All-E-RR-zeaxanthin configuration predominates in the human serum. The average percentage of the tentatively identified RS- configuration has been calculated and is $3.37 \%$. However, the percentage of tentatively identified SS- confirmation is generally below the detectable level. The result obtained from this study indicates that the RS and SS zeaxanthin isomers in the retina are probably formed by the metabolism of lutein in the retina.



Figure 40. HPLC chromatogram of dicabamated zeaxanthin racemic-mixture standard on a Normal phase HPLC 


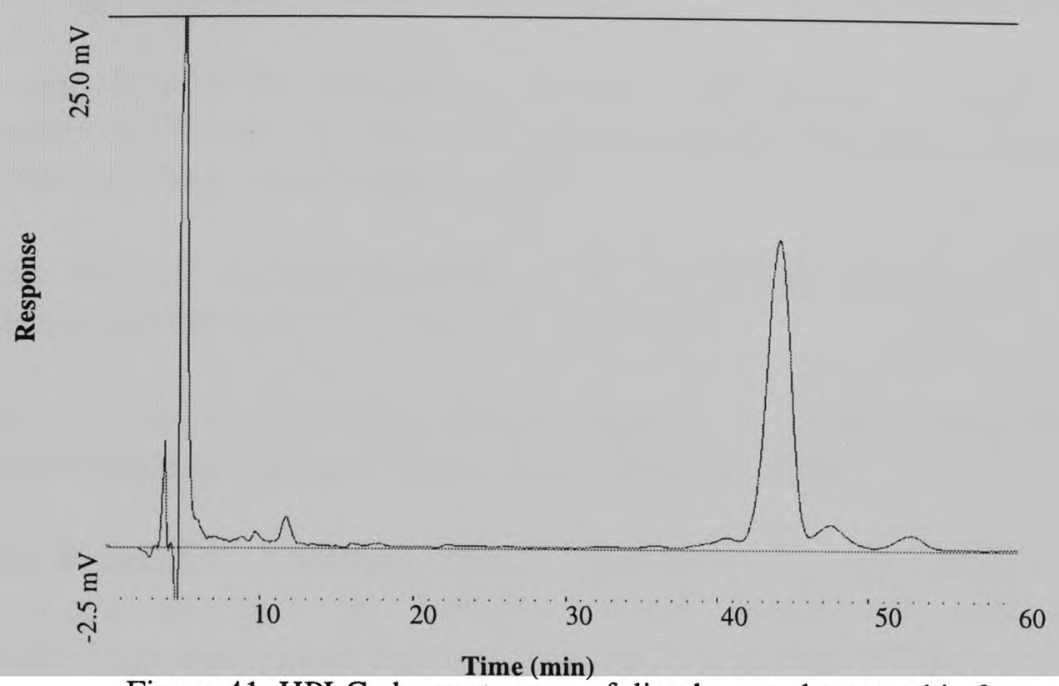

Figure 41. HPLC chromatogram of dicarbamated zeaxanthin from serum extract on a Normal phase HPLC

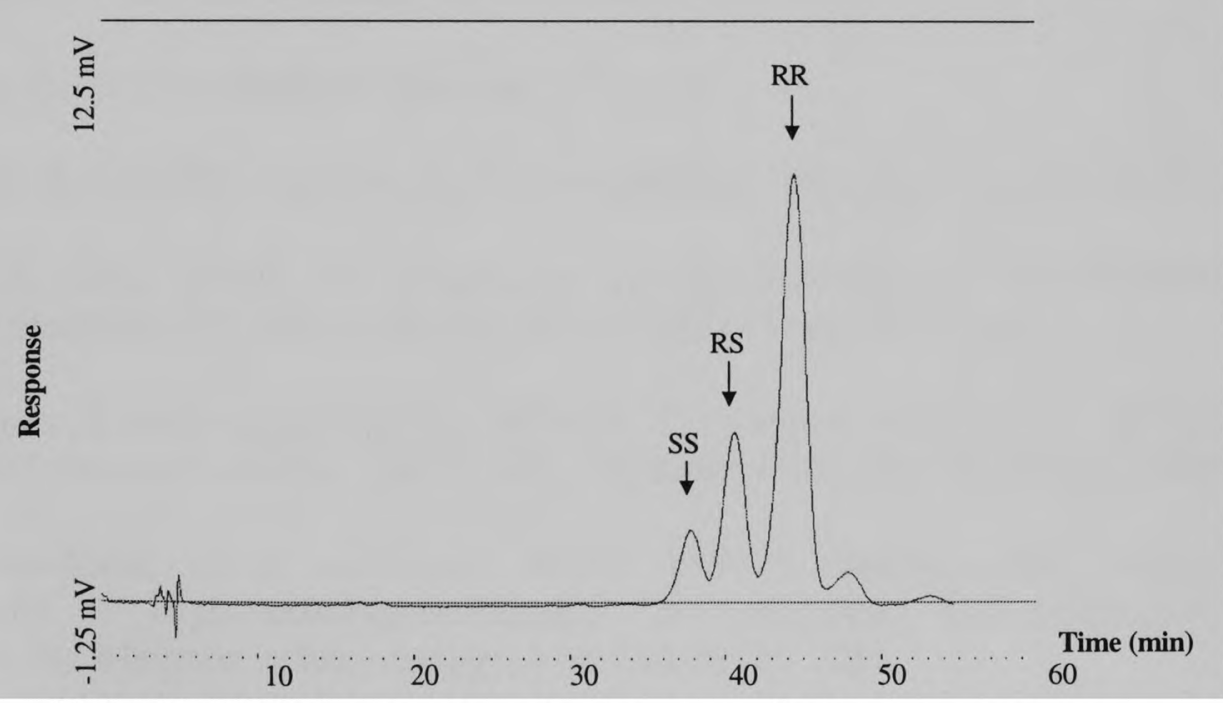

Figure 42. HPLC chromatogram of co-injection collected peak in a elution of dicarbamated zeaxanthin from serum extract with dicarbamated zeaxanthin racemic-mixture standard on a normal phase HPLC 


\section{List of References}

1. Barua, Arun B., and Furr, Harold C. (1992), Extraction and Analysis by HighPerformance Liquid Chromatography of Carotenoids in Human Serum, in: Methods in Enzymology. Vol. 213, (Lester Packer, Ed) Pp.273-281. Academic Press, San Diego.

2. Bowen, Phyllis E., Mobarhan, Sohrab. and Smith, J. Cecil. (1993), Carotenoid Absorption in Humans in: Methods in Enzymology. Vol. 214, (Lester Packer, Ed) Pp.317. Academic Press, San Diego.

3. Bone, Richard A., and Sparrock, J. M. B. (1971), Comparison of macular pigment densities in human eyes. Vis. Res. 11, 1057-1064.

4. Bone, Richard A, Landrum, John T, Tarsis S. L. (1985), Preliminary identification of the human macular pigment. Vision Res. 25: 1531-1535.

5. Bone. Richard A., Landrum, John T., Fernandez, L., and Tarsis, S. (1988), Analysis of the macular pigment by HPLC: Retinal distribution and age study, Invest. Ophthalmology and Visual Science. Vol. 29, No. 6. June 1988.

6. Bone Richard A., Landrum, John T., and Cains, A. (1992), Optical density of the macular pigment in vivo and in vitro. Vis. Res. 32, 105-110.

7. Bone. Richard A., Landrum, John T., Friedes, L., Gomez, C. M., Kilburn, M., Menendez, E., Vidal, I., Wang, W. (1997), Distribution of lutein and zeaxanthin stereoisomers in the human retina Exp. Eye Res. 64, 211-218.

8. Britton, G., (1985), Methods Enzymol., 111, 113.

9. Cogdell, R. J. (1985), Carotenoids in Photosynthesis. Pure Appl. Chem. 57, 723-728.

10. Davies, B.H., (1976), In "Chemistry and Biochemistry of Plant Pigments", $2^{\text {nd }}$ Edition (Goodwin. T.W.,Ed,) Vol. 2, P.38, Academic Press, New York.

11. De Ritter, E. and Purcell, A. E. (1981), In "Carotenoids as Colorants and Vitamin A Precursors" (Bauernfeind. J.C., Ed,) P.815, Academic Press, New York and London.

12. De Leenheer, A. P., and Nelis, H. J. (1992), Profiling and Quantitation of Carotenoids by High-Performance Liquid Chromatography and Photodiode Array Detection. In: Methods in Enzymology. Vol. 213. Pp.251-265.

13. Eye Disease Case-Control Study Group. (1993), Antioxidant status and neovascular age-related macular degeneration. Arch. Ophthalmol. 111, 104-109. 
14. Handelman, G. J., Snodderly, D. M., Krinsky, N. I., Russett, M. D., and Adler, A. J., (1991), Biological control of primate macular pigment; biochemical and densitometric studies. Invest. Ophthalmol.Vis. Sci. 32, 257-67.

15. Handelman, Garry J., Shen, Binghua, and Krinsky, Norman, I. (1992), High Resolution Analysis of Carotenoids inHuman Plasma by High-Performance Liquid Chromatography. In: Methods in Enzymology. Vol. 213. Pp. 336-346.

16. Handelman, G. J., Max Snodderly, D., Adler, A. J., Russett, M. D., and Dratz, E. A., (1992), Measurement of carotenoids in human and monkey retinas In: Methods in Enzymology. Vol. 213. Pp. 220-230.

17. Hyman, L. (1992), Epidemiology of AMD. In “ Age-related Macular Degeneration : Principles and Practice" (G.R. Hampton and D.T. Nelson, eds.), pp. 1-35. Raven Press, New York.

18. Khachik, Frederick, Beecher, Gary R., Goli, Mudlagiri B., and Lusby, William R., (1992), Separation and Quantitation of Carotenoids in Foods, in: Methods in Enzymology. Vol. 213, (Lester Packer, Ed) Pp. 347-359. Academic Press, San Diego.

19. Khachik, Frederick, Beecher, Gary R., Goli, Mudlagiri B., Lusby, William R., Smith, James C. (1992). Separation and identification of carotenoids and their oxidation products in the extracts of human plasms. Anal. Chem. 64, 2111-2122.

20. Khachik, Frederick, Engkert, Gerhard, Beecher, Gary R., Smith, J. Cecil. (1995), Isolation, structural elucidation, and partial synthesis of lutein dehydration products in extracts from human plasma. J Chromatog. Bio. 670, 219-233.

21. Khachik, Frederick, Spangler, Christopher J., Smith, J. Cecil. (1997), Identification, quantification, and relative concentrations of carotenoids and their metabolites in human Milk and serum. Anal. Chem. 69. 1873-1881.

22. Khachik, Frederick, Bernstein, Paul S., Garland, Donita L. (1997), Identification of lutein and zeaxanthin oxidation products in human and monkey retina. Invest. Ophthalmol. Vis. Sci. 39, 1802-1811.

23. Khachik, Frederick, Beecher, Gary R., Goli, Mudlagiri B., Lusby, William R. and Daitch, Charles E. (1992), Separation and Quantification of Carotenoids in Human Plasma, in: Methods in Enzymology. Vol. 213, (Lester Packer, Ed) Pp.205-219. Academic Press, San Diego.

24. Khachik, Frederick, Beecher, Gary R., and Smith, J. Cecil, Jr., (1995), Lutein, Lycopene, and Their Oxidative Metabolites in Chemoprevention of Cancer. J. of Cellular Biochemistry, Supplement 22: 236-246 
25. Kohler, B. E. (1995), Carotenoids Volume 1B: Spectroscopy (Britton. G., LiaaenJensen. S., Pfander. H., Ed,) Birkhauser Verlag, Basel.

26. Koyama, Yasushi et al. (1990), Configurations of Carotenoids in the Reaction Center and the Light - Harvesting Complex of Rhodospirillum rubrum. Natural Selection of Carotenoid Configuretions by Pigment Protein Complexes. Photochemistry and photobiology Vol. 51, No. 1, pp. 119-128.

27. Landrum, John T., Bone, Richard A., Vidal, T., Menendez, E., and Kilburn, M. (1995), Macular pigment stereoisomers in individual eyes: A comparison between normals and those with age-related macular degeneration. Invest. Ophthalmol. Vis. Sci. $19,875-863$.

28. Landrum, John T., Bone, Richard A., Kilburn, M. D. (1997), The macular pigment: A possible Role in Protection from Age-Related Macular Degeneration. Advances in Pharmacology, Vol. 38 pp. 537-556.

29. Landum, John T., Bone, Richard A., Joa, Hilda, Kilburn, Mark D., Moore, Linda 1. and Sprague, Kathleen E. (1997), A one year study of the macular pigment: The effect of 140 days of a lutein supplement. Exp. Eye Res. 65, 57-62.

30. Landrum. John T., Bone, Richard A., Moore, L. L., Gomez, C. M. (1999), Analysis of zeaxanthin distribution within individual human retinas, Methods in enzymology. Vol 299 ,

31. Neal, E. Craft. (1992), Carotenoids Reversed-Phase High-Performance Liquid Chromatography Methods: Reference Compendium. In: Methods in Enzymology. Vol. 213. Pp.185-205.

32. Pfander, Hanspeter. (1992), Carotenoids: An Overview, in: Methods in Enzymology. Vol. 213, (Lester Packer, Ed) Pp. 3-13. Academic Press, San Diego.

33. Schalch, W. (1992), Carotenoids in the reteina- A review of their possible role to prevent or limit damage caused by light and oxygen. In: Emerit BI, Chance B, eds. Free Radicals and Aging. Basel: Birkauser Verlag; 280-298.

34. Seddon, J. M., Ajani, U. A., Sperduto, R. D., Hiller, R., Blair, N., Burton, T. C., Farber, M. D., Gragoudas, E. S., Haller., J., Miller, D.T., Yannuzzi, L.A., and Willett, W. (1994), Dietary carotenoids,vitamins A, C, and E and advanced age-related macular degeneration. JAMA 272, 1413-1420.

35. Sowell, Annel., Huff, Daniel L., Gunter, Elaine W., and Driskell, Willuam J. (1988), Idetification of cis-carotenoids in human sera analyzed by reversed - phase high -performance liquid chromatography with diode array detection. Journal of Chromatography, 431, 424-430. 
36. Sprague, Kathleen E. (1997), Quantification and metabolism of lutein, zeaxanthin and their oxidation products. M.S Thesis, Florida International University.

37. Straub, Otto. (1987), Key to Carotenoids. $2^{\text {nd }}$ ed. Pfander, H., Ed. Birkhauser Verlag. Basel.

38. Strohschein, S., Schlotterbeck, G., Richter, J., Pursch, M., Tseng, L.-H., Handel, H., Albert, K. (1997), Comparison of the separation of cis / trans isomers of tretinoin with different stationary phases by liquid chromatography-nuclear magnetic resonance coupling. Journal of Chromatography A, 765, 207-214.

39. van Breemen, Richard B., Schmitz, Harold H., and Schwartz, Steven J. (1993), Continuous-Flow Fast Atom Bombardment Liquid Chromatography/Mass Spectrometry of carotenoids. Analytical Chemistry, 65, 965-969.

40. van Breemen, Richard B. (1996), Innovations in Carotenoid Analysis Using LC/MS. Analytical Chemistry News \& Features. 299A-304A.

41. Zechmeister, L. (1962), cis-trans Isomeric Carotenoids Vitamins A and Arylpolyenes. 